\title{
Investigation of the Rayleigh-Taylor and Richtmyer-Meshkov instabilities
}

\author{
Research Funded under the \\ Stewardship Science Academic Alliances Program \\ DOE Grant ID Number: DE-FG52-03NA00061
}

\author{
Riccardo Bonazza, Mark Anderson and Jason Oakley \\ Department of Engineering Physics \\ University of Wisconsin - Madison \\ Final scientific report for the period 11/2002 - 3/2006
}

\section{Executive summary}

The program focuses on the experimental and numerical study of two instabilities that develop at the interface between two different fluids when the interface experiences an impulsive or a constant acceleration. The instabilities, called the Richtmyer-Meshkov and Rayleigh-Taylor instability, respectively (RMI and RTI), adversely affect target implosion in experiments aimed at the achievement of nuclear fusion by inertial confinement by causing the nuclear fuel contained in a target and the ablated shell material to mix, leading to contamination of the fuel, yield reduction or no ignition at all.

Specifically, our work has been articulated in three main directions: study of impulsively accelerated spherical gas inhomogeneities; study of impulsively accelerated 2-D interfaces; study of a liquid interface under the action of gravity. Two main objectives were pursued in our activities: i) to advance the current understanding of the physics of these instabilities, with particular emphasis on the effect that the strength of the accelerating shock and the density contrast across the two gases have on the impulsively accelerated interfaces; ii) and to develop a database at previously untested conditions to be used to calibrate and verify some of the computational tools being developed within the RTI/RMI community at the national laboratories and the ASCI centers.

The program has yielded important results on all fronts. Extensive experimental series have been performed to study shock-accelerated bubbles containing several different gases. An entirely new apparatus for the preparation of a bubble, its release in the shock tube and its pre- and post-shock imaging was designed, fabricated and installed in the main shock tube facility. Planar imaging of shock-accelerated bubbles was performed, for the first time, at shock strengths of up to $M=3$. Flow details that had not been resolved in previous experiments using different imaging techniques were observed and understood for the first time. A very high level of integration has been developed between the experiments and very extensive numerical calculations performed using the Raptor code made available by Lawrence Livermore National Laboratory. Experiments were designed and interpreted through the calculations and the experimental results provided a high degree of validation for the calculations. 
An entirely new apparatus for the preparation of a two-dimensional interface with perturbations described by fewer than 10 modes was also designed, fabricated and installed in the main shock tube facility. Experiments were performed for the first time to observe the behavior of the interface after acceleration by a shock of strength $M>2$ and preliminary Raptor calculations under the same conditions were performed.

An entirely new experiment was developed for the study of the RTI of the interface between two liquids under the action of gravity. The experiment is based on the use of magnetorheological fluids, viz. fluids that can be frozen into a desired shape by the application of a sufficiently strong magnetic field. The experiment has undergone many revisions and improvements while yielding a first database of results in the process.

All three experimental setups and their respective diagnostic techniques have now been successfully operated and new data have been generated from each of them. All these outcomes are an integral part of the nation's effort towards the achievement of controlled nuclear fusion by means of inertial confinement for the purpose of energy production. 


\section{General background}

When a light fluid is continuously accelerated against a heavy one, the amplitude of any perturbations on the interface separating the two fluids grows unboundedly, leading to the distortion and rupture of the interface and the consequent mixing of the two fluids. This phenomenon is known as the Rayleigh-Taylor instability and it is a consequence of the baroclinic generation of vorticity due to the non-zero cross product between the misaligned pressure and density gradients at the interface between the two fluids.

The Richtmyer-Meshkov instability occurs if the interface between the fluids is accelerated impulsively rather than continuously (e.g. by a shock wave). In this case, the mismatch in acoustic impedance and the misalignment between the pressure and density gradients lead to both a refraction of the shock and the generation of vorticity at the interface, which in turn leads to the growth of the perturbations and the interface distortion. If the shock travels from the hard (high acoustic impedance) into the soft fluid, an initial phase reversal takes place, with peaks and troughs trading places along the interface, followed by the unbounded growth of the perturbations.

The RT and RM instability occur at both very large length scales, e.g. in astrophysical settings, and very small scales like in the case of inertial confinement fusion (ICF) experiments. In this latter case, the microtarget containing the $\mathrm{D}$ - $\mathrm{T}$ fuel, to be imploded with a laser-generated shock wave in a facility such as the Omega or NIF, inevitably contains manufacturing imperfections (surface perturbations) that grow upon ablation and subsequent acceleration, leading to the mixing of the shell material with the nuclear fuel, the fuel's contamination and a reduction in the reaction's thermonuclear energy yield. Indeed, the two instabilities often both arise in an ICF experiment with one or the other being the dominant depending on the shape of the laser pulse. Thus, an understanding of the initial, intermediate and late stages of these instabilities, of the physical mechanisms responsible for the onset and evolution of the distortion and disruption of the initial interface shape, and of the consequences of these events (turbulence, fluid mixing) is one of the essential requirements of the ICF effort.

Our experiments are performed in a vertical, downward-firing, square shock tube of large cross section (to minimize the influence of wall effects on the behavior of the fluid interface) and high structural capability (for the generation of strong shocks without the need to operate at very low initial pressure).

In the original proposal, experiments to study both the RTI and the RMI had been planned around the experimental setup that had been developed and utilized during the previous funding cycle of the present program. That involved a retractable plate, with a sinusoidal perturbation imposed along one of its dimensions, to initially separate two gases of different density, the heavier gas being above the plate. The experiment would begin with the retraction of the plate out of the shock tube, leaving a sinusoidal, diffuse interface to evolve under the action of gravity. Since the heavy gas was on top of the light one, the RTI would onset causing the interface perturbation to amplify and eventually distort into the well known mushroom patterns and into full mixing of the two gases. This process could be used either to study the development of the RTI itself (no shock wave released) or to form the initial condition for an RMI experiment (a shock wave would accelerate the interface when its shape was still sinusoidal). The fundamental hypothesis was that this procedure would generate two-dimensional flows in both cases. 
Unfortunately, characterization of the interface after plate retraction, by imaging it in two vertical planes, perpendicular and parallel to the direction of plate retraction, demonstrated that the flow in the wake behind the plate caused strong three-dimensional effects that would have been very hard to quantify and thus would have made the interface unsuitable as an initial condition for both the RTI and RMI experiments. New experiments had therefore to be designed for both studies.

In the RTI case, the experiments were shifted from gas to liquid interfaces and magnetorheological fluids were chosen for their property of "freezing" into almost any shape under the action of a magnetic field. For the RMI, two new separate experiments were designed, for the study of twodimensional and three-dimensional interfaces, respectively.

In the original proposal, it had been envisioned that numerical experiments similar to the laboratory ones would be performed using the $S A G E$ code that was going to be made available by colleagues at Los Alamos National Laboratory. Unfortunately, no access to the $S A G E$ could be obtained but the Raptor code was made available by Lawrence Livermore National Laboratory and very extensive calculations have since been performed with it.

\section{Experimental apparatus}

\subsection{Shock tube facility}

The experiments for the study of impulsively accelerated interfaces are performed in a vertical, square shock-tube which has now been operated successfully for nine years. The tube was designed with two main objectives in mind: minimize the effect that the boundary layers that develop behind the incident shock have on the behavior of the interface, after the initial impulsive acceleration; and to allow for the use of strong shocks (up to $M=5$ ) into a driven and a test gas initially at atmospheric pressure (since lowering the initial gas pressure, to maintain the post-shock pressure below the structural capability of a weaker tube, causes the kinematic viscosity of the gases, and the thickness of the boundary layers, to increase, with adverse effects on the visibility of the interface).

The shock-tube is vertical, with large $\left(25 \times 25 \mathrm{~cm}^{2}\right)$ square inner cross section, and approximately $9 \mathrm{~m}$ long. The structure can withstand a $20 \mathrm{MPa}$ pressure load. The square cross section provides the parallel walls necessary for flow visualization without resorting to a structurally weak tube extension. The tube is vertical to make use of gravitational stratification in preparing a continuous interface between gases of different densities. Sketches of the tube assembly and its cross section are shown in Fig. 1.

The driver section is made from circular, chrome-plated, carbon steel pipe, $38 \mathrm{~cm}$ ID. The inside of the driven section of the tube is a square liner consisting of four stainless steel plates welded together; the liner is supported by a concrete matrix contained in a circular carbon steel pipe.

The driver section is at the top of the tube; the whole assembly is supported by three cylindrical legs, an in-ground $2 \mathrm{~m}^{3}$ concrete foundation and a square tubing support structure. The shock tube is evacuated prior to introducing the driven and test gases to maintain high purity of the individual species.

Two $0.05 \mathrm{~m}^{3}$ boost tanks are connected to the driver section via fast opening valves. During an experimental run, the driver section is pressurized to $90 \%$ of the diaphragm rupture pressure and 

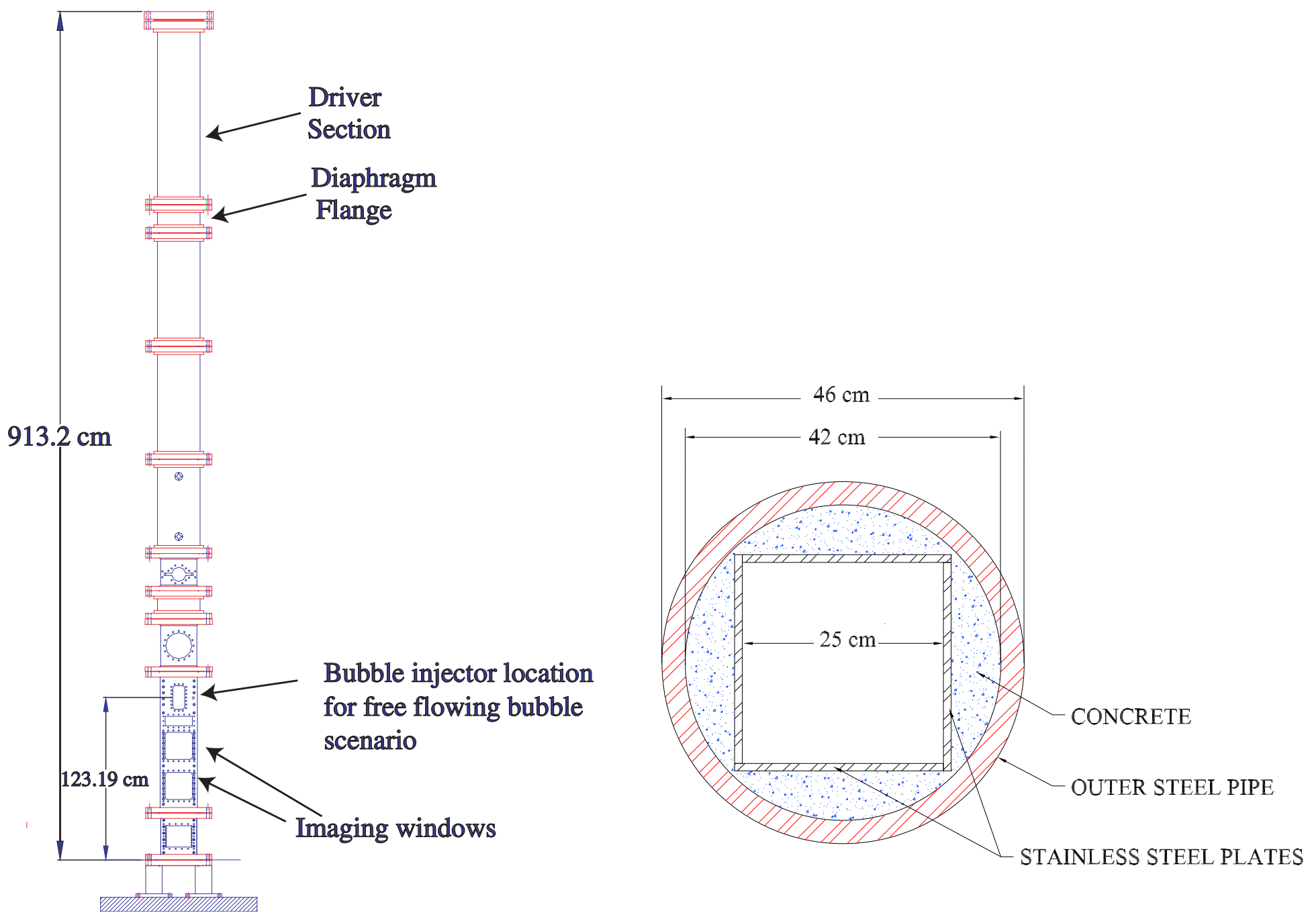

Figure 1: Schematics of the shock-tube facility and its cross section.

the boost tanks are pressurized to a level sufficient to drive a choked flow into the driver once the fast acting valves open. An extensive empirical database has been developed about the time that elapses between the issue of the boost tank valve-opening command and the rupture of the diaphragm, thus acquiring excellent control over the time of shock release.

A new test section was designed and fabricated during this funding period; the new chamber contains the openings necessary to mount the interface-forming hardware (both for the bubble and the 2-D sinusoid), 12 openings for imaging and instrumentation mounting. A view of the test section is shown in Fig. 2.

The tube end wall also allows for optical access through a circular window, $14 \mathrm{~cm}$ in diameter, centered $7.5 \mathrm{~cm}$ from the tube's inner wall so that a laser sheet can be shone upward at various distances from the tube's center line.

Typically, the driver gas is either helium or nitrogen while the driven gas is either nitrogen or argon, depending on: desired shock strength; acceptable speed of the rarefaction wave that develops upon 


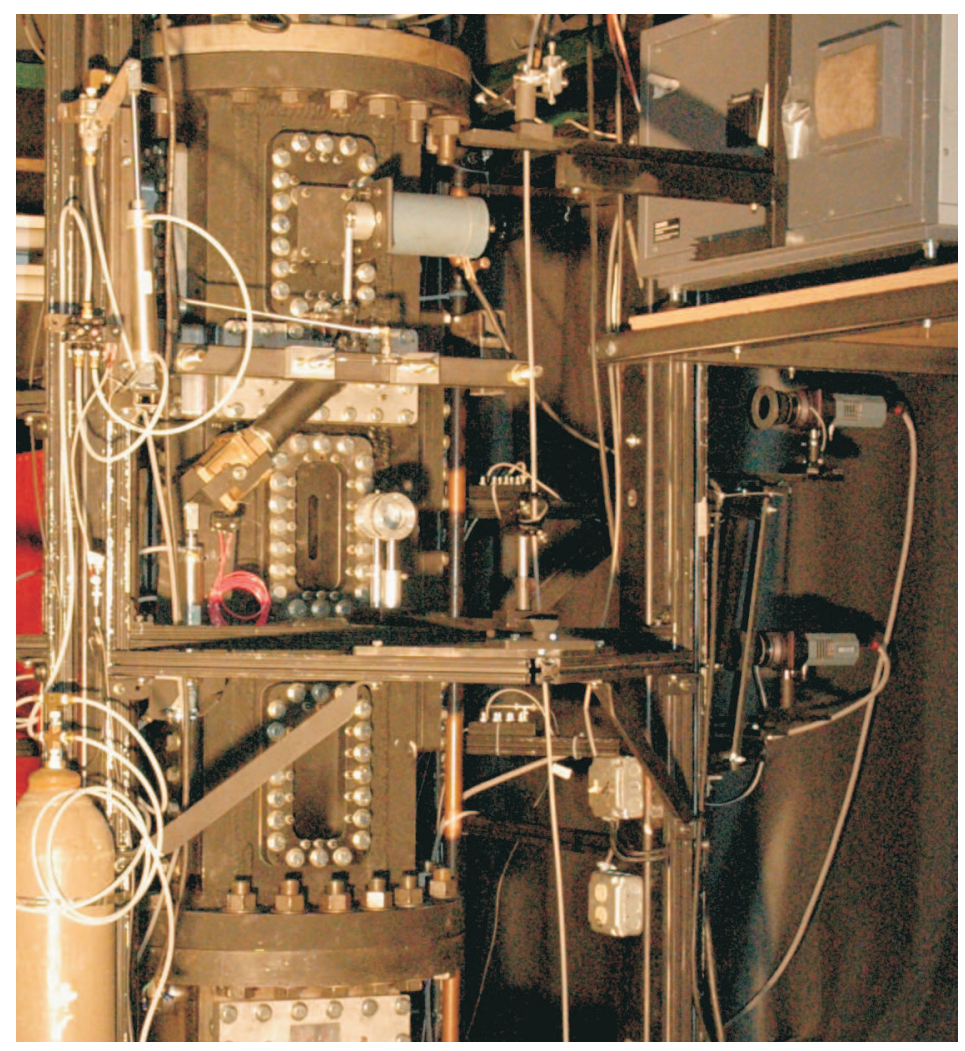

Figure 2: Shock tube test section.

rupture of the diaphragm; and maximum pressure resulting in the driven section.

\subsection{Instrumentation}

A large instrumentation pool is available for all the experiments, including:

1. three pulsed excimer lasers

2. one pulsed Nd:YAG laser

3. two CW Ar-ion lasers

4. three scientific grade, back-lit, UV-sensitive, 1024× 1024 CCD cameras

5. one scientific grade, back-lit, $1024 \times 1024$, CCD camera

6. two high-speed (260 fps), medium resolution $(512 \times 512)$ CCD cameras

7. three PC-based data acquisition platforms

8. two PC-based digital control systems 


\section{Accomplishments}

\subsection{Impulsively accelerated spherical inhomogeneities}

\subsubsection{Background}

Both cylindrical and spherical gas inhomogeneities subjected to impulsive acceleration had been studied in the past. Theoretical analyses and numerical investigations had shown that, upon shock acceleration, a spherical inhomogeneity initially distorts simply because of compression and later develops a vortex ring around its equatorial plane due to the vorticity produced by the misalignment of the density and pressure gradients. The most relevant flow parameters previously investigated [1] included the velocity deficit of the inhomogeneity with respect to the surrounding flow; the circulation of the vortex ring. The relevant dimensionless numbers are the Mach number of the accelerating shock and the density contrast expressed as the Atwood number, $A=\left(\rho_{1}-\rho_{2}\right) /\left(\rho_{1}+\rho_{2}\right)$.

Previous shock tube experiments had been performed at different institutions with cylindrical interfaces prepared with nitrocellulose membranes or as free laminar jets; and with spherical interfaces consisting of soap bubbles filled with a test gas. All the previous experiments had been performed in horizontal shock tubes at low Mach numbers $(\leq 1.5)$. The bubbles were held in place by small injectors protruding slightly inside the shock tube. Integrated, line-of-sight (schlieren) and planar (planar laser induced fluorescence) imaging techniques had been employed to visualize the flows.

\subsubsection{Laboratory experiments}

In the present experiments, a soap bubble containing a test gas (either heavier or lighter than air) is generated at the tip of an injector and then released (either in free-fall or free-rise) into the shock tube, while the injector retracts into the shock tube wall so as not to obstruct the incoming shock wave and the ensuing flow. An image of the injector and a schematic of the imaging setup are shown in Figs. 3 and 4, respectively.

The bubble is imaged during the initial stages of its free fall using continuous, infrared, front lighting and a medium resolution $(512 \times 512$ pixel $)$ medium framing rate $(260 \mathrm{fps}) \mathrm{CCD}$ camera. After being accelerated by the shock, the bubble is imaged at three different time delays: once by simultaneous shadowgraphy and Mie scattering planar imaging; and twice more by planar imaging. The shadowgraph illumination is provided by a flashing white source while planar imaging is performed

with either a double-pulse Nd:YAG laser at $532 \mathrm{~nm}$ or an excimer laser at $248 \mathrm{~nm}$. In planar Mie scattering, the laser light is scattered from the shocked bubble towards a CCD sensor by the soap film which atomizes upon shock acceleration and thereafter acts as a suitable flow-tracer. The size of the atomized droplets depends on the shock strength. The average droplet size is estimated to be on the order of $1 \mu \mathrm{m}$, for a bubble accelerated by a $M=2.88$ shock [2,3]. At lower Mach number, it may be noted that, because of the lower energy deposited by the shock on the bubble film, the atomization is less efficient. The average droplet size is estimated on the order of $30 \mu \mathrm{m}$, for a bubble accelerated by a $M=1.33$ shock.

The collimated beam used for the shadowgraph image propagates in a direction orthogonal to the laser sheets used for planar imaging. All four post-shock images are recorded on high resolution $(1024 \times 1024$ pixels $)$ scientific grade CCD cameras: the shadowgraph image on one sensor; the two 


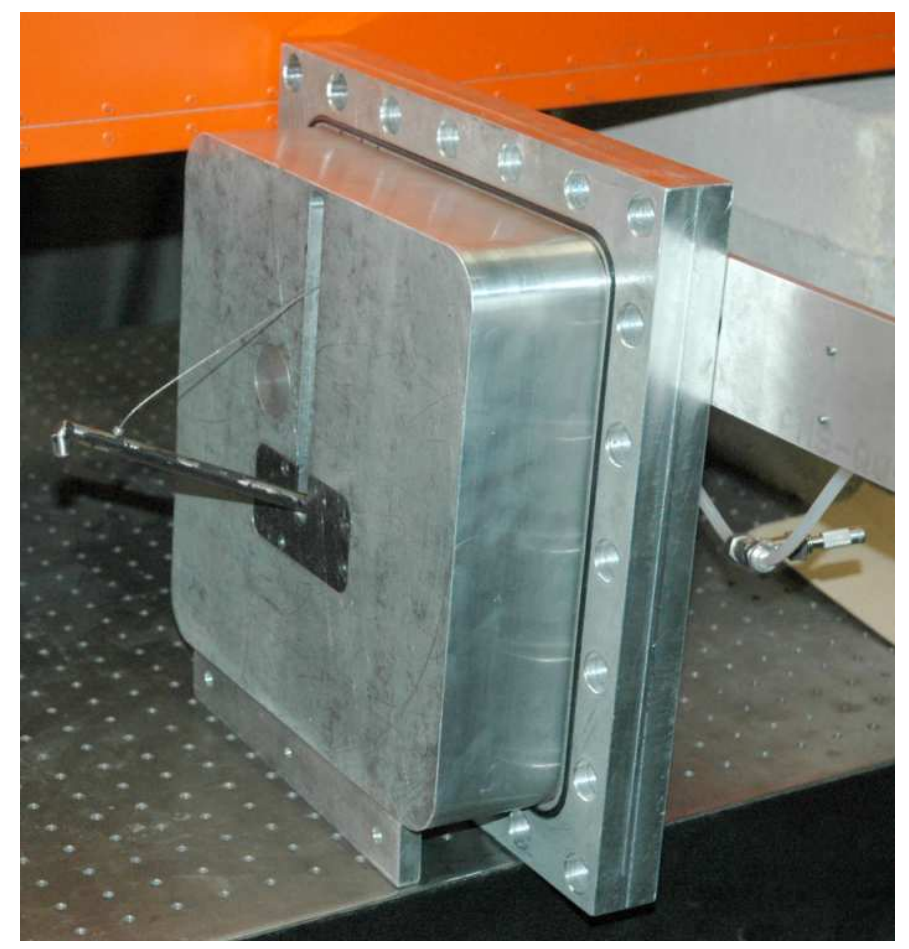

Figure 3: Retracting injector.

planar views from the YAG double pulse on a second sensor; and the planar image from the excimer laser on a third sensor. Since the shadowgraph and the first of the two YAG images are recorded at $90^{\circ}$ from each other at the same time delay (and thus the same position) the cross section of the laser sheet appears as a bright line in the shadowgraph image and determines the position of the bubble relative to the sheet. This is important when comparing experimental to computational images in that it provides precise knowledge of which bubble cross section is being observed in the laboratory.

At all shock strengths, the following sequence takes place: the bubble is initially distorted because of pure compression; the vorticity baroclinically deposited on the interface then leads to the formation of a vortex ring seen in cross section at the left and right sides of the bubble; in turn, the vortex ring induces very high strain on the top surface of the bubble, causing it to tilt upwards. A secondary vortex ring sometime develops at the top of the bubble (details about its origin are given in Sec. 3.1.3 below); in some cases, a jet protrudes above the secondary mushroom.

The height and width of the bubble, the major and minor diameters of the primary vortex ring, and the velocity of the vortex ring are all measured directly the images.

Different approaches have been tried to determine the appropriate normalizing quantities for the bubble macroscopic geometrical features and the post-shock time when images are recorded. The best results are obtained normalizing both the height and the width by the initial bubble diameter $D$; and the post-shock time by $\tau=D / W^{*}$, where $W^{*}$ is the velocity of the faster between the shock transmitted into the bubble and the shock traveling outside the bubble.

The experimental results are discussed in detail in Secs. 3.1.4 and 3.1.5 together with the corre- 


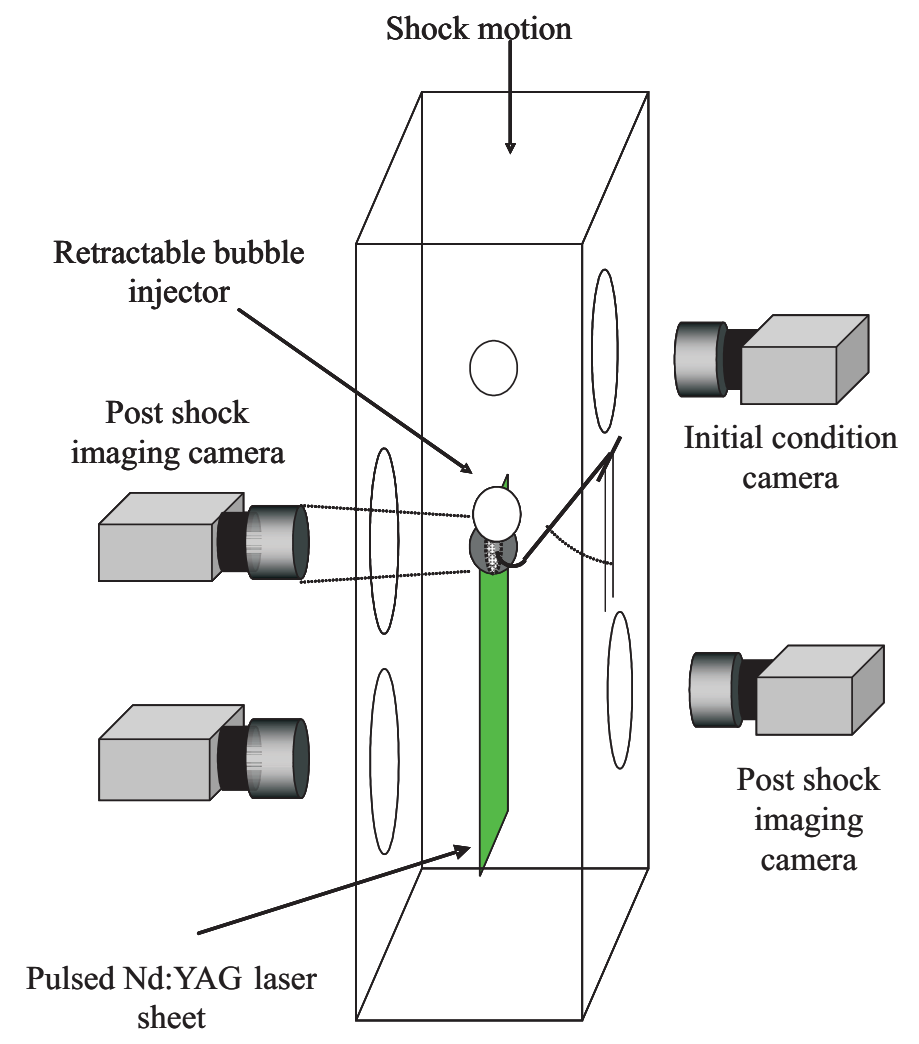

Figure 4: Experimental setup to capture multiple bubble images per run.

sponding numerical simulations.

\subsubsection{Computational experiments}

Extensive computational experiments have been performed using the Raptor code made available by Lawrence Livermore National Laboratory. One of our graduate students and one of our scientists have worked very closely from here at UW with Dr. Jeff Greenough of LLNL; the graduate student also spent twelve weeks for each of two consecutive summers at LLNL on an internship, also performing computational runs simulating the laboratory experiments. 2-D calculations were initially performed assuming axial symmetry. 3-D runs over a quarter of the sphere have now been performed for a total of 12 different scenarios (combinations of Mach and Atwood numbers). The reason for the 3-D calculations is that azimuthal (Widnall-type) instabilities render the 2-D runs inadequate.

To avoid the stairstepping effects consequent to the presence of a round object (the bubble) within a square grid, a new film-modeling technique was developed: since the grid resolution is much larger than the soap film thickness $(\sim 1 \mu \mathrm{m})$, a spherical soap film layer with thickness equal to one cell width is placed in the domain, with a uniformly scaled density to match the actual total film mass used in the laboratory. Sub-cell sampling is then used to determine the amount of soap present within each cell. 


\subsubsection{Convergent geometry, low density contrast}

Figure 5 shows the evolution of the shocked bubble after contact with a $M=2.88$ shock with a wave velocity $W_{i}=1017 \mathrm{~m} / \mathrm{s}$, an $N_{2}$ particle velocity $u_{1}^{\prime}=745 \mathrm{~m} / \mathrm{s}$ and transmitted shock wave speed $W_{t}=993 \mathrm{~m} / \mathrm{s}$. The time is non-dimensionalized as $\tau=t / \tau *$. The characteristic time $\tau *=D /\left(2 W_{t}\right)$, is defined as the cloud crushing time, where $D$ is initial diameter of the bubble. Several experiments have been conducted and the reproducibility between different experiments is quite good with bubble diameters in the range $D=5.0 \pm 0.02 \mathrm{~cm}$ and incident Mach numbers in the range $2.88 \pm 0.04$.

In Fig. 5(a), the shock is visible midway through its transit across the argon bubble region. Bright points at the upper and lower limbs of the bubble indicate the upper and lower edges of the initial bubble film. The film layer is atomized upon shock impact, and microscopic particles of liquid film are entrained nearly uniformly into the flow behind the transmitted shock. The refractive effect of the heavier gas introduces a slight upward bow into the shock front, and the longitudinal compressive effect of the shock flattens the upper portion of the bubble. Shock refraction is complete, and the refracted shock has passed just downstream of the bubble, in Fig. 5(b).

Figure $5(c)$ shows the bubble when the shock is approximately 1.5 diameters downstream and the bubble has been compressed into an inverted bowl-type shape. The strongest vorticity deposition predicted by the baroclinic model is at the left and right bubble limbs, and at these locations, in Figs. 5(d)- $(f)$, a primary vortex ring (hereafter referred to as PVR) can be seen developing at the downstream end of the bubble.Furthermore, the small scales introduced into the vorticity field by the presence of the film material lead to a number of secondary features that can be seen forming on the bubble surface at this time. The presence of film leads to the formation of the reflected shock wave from the downstream end of the bubble. The upper surface of the bubble is becoming distorted and a secondary vortex ring (hereafter referred to as SVR) associated with the appearance of an upper mushroom structure is becoming evident. The dominant feature seen in Figs. $5(e)-(f)$ is the PVR at the downstream end of the bubble. A small SVR is also observed at the upstream end of the bubble. The SVR at the upstream surface has the same sense of rotation as the PVR at the downstream surface of the bubble. The PVR entrains ambient fluid from the center portion of the image and swirls it into the center of the vortex ring.

In Fig. 5(e), along with the PVR and the SVR, a secondary jet is evident at the top of the image. The secondary jet and vortex ring on the upstream surface of the bubble represent the combined effect of nonuniformities on the initial bubble film and of the passage of the internally reflected shock over the upstream surface of the bubble. In particular, liquid film mass accumulated at the bottom of the initial bubble film in a gravitational meniscus is ejected in the upstream direction after shock passage, forming a jet which pierces the upstream surface of the bubble. This leads to the growth of additional vortical features near the apex and upper shoulders of the bubble. At the same time, a reflected shock wave moves upstream across the argon region from the interior bottom surface of the bubble, and, upon crossing the upstream surface of the bubble, induces the further growth of secondary vortices as shown numerically by Zabusky et al. in 1998 [4] but never confirmed experimentally.

The bubble asymmetry about the vertical axis observed in Fig. $5(f)$ is due to the onset of the bending mode instability, commonly known as Widnall instability [5] for laminar flows. One can

also observe that the PVR is connected at the top of the image through a cone-shaped channel 


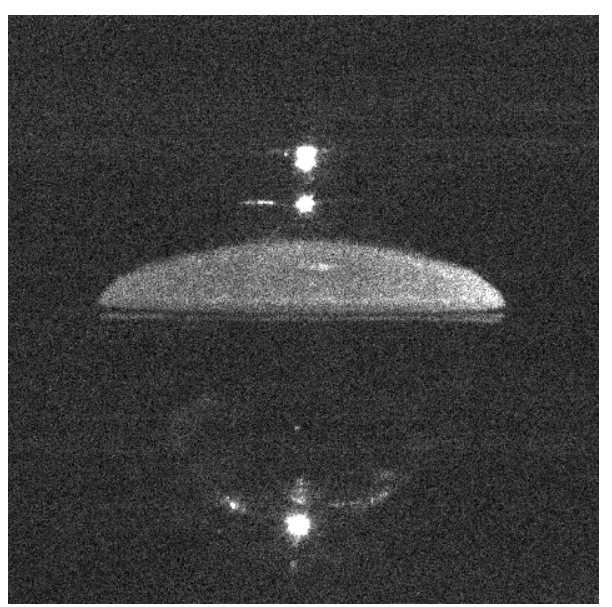

$(a)$

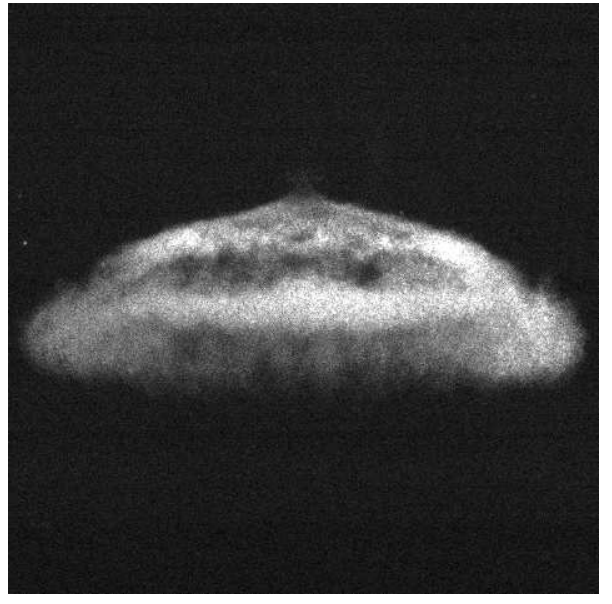

$(c)$

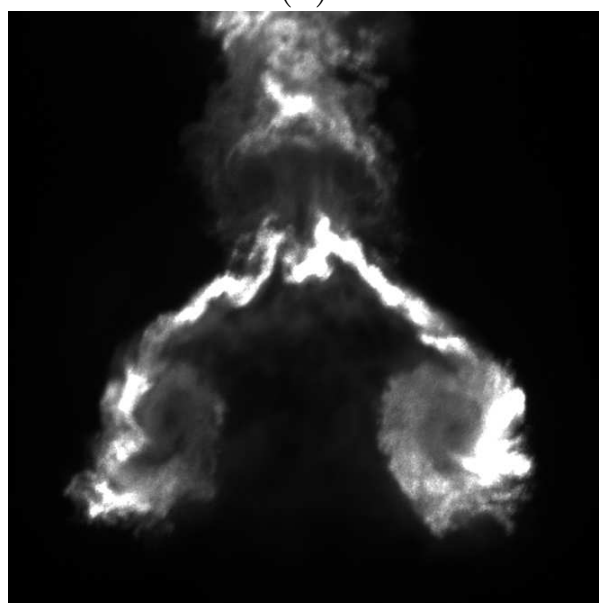

$(e)$

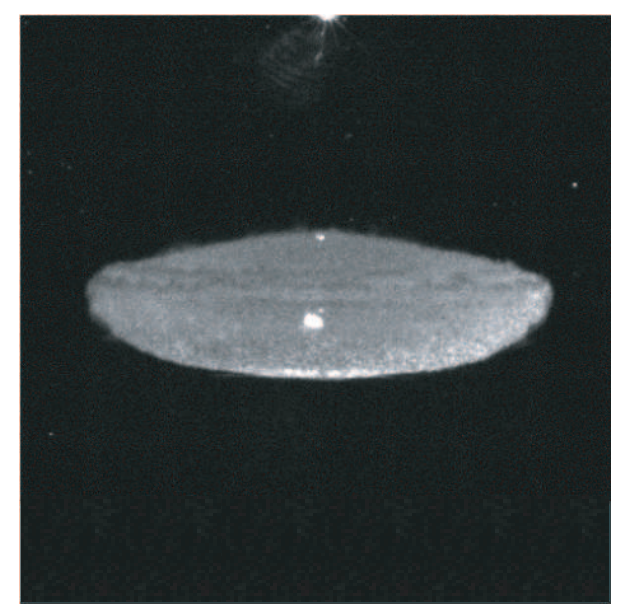

(b)

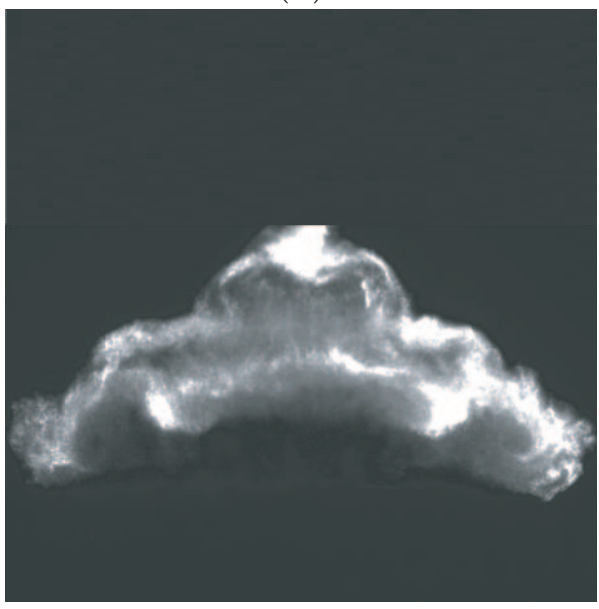

$(d)$

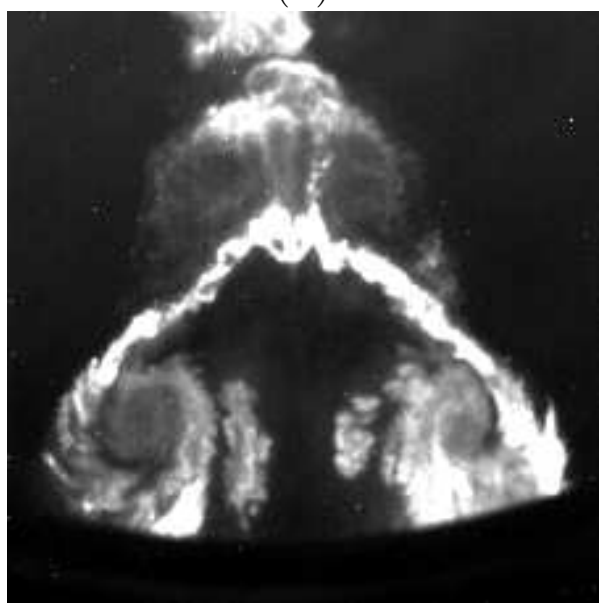

$(f)$

Figure 5: Experimental images of planar laser light Mie-scattered near the bubble midplane, at time $\tau$ after the initial interaction of a $M=2.88$ shock wave with the bubble: $(a) \tau=0.92,(b)$ $\tau=2.02,(c) \tau=6.61,(d) \tau=10.91,(e) \tau=21.08,(f) \tau=21.30$. 
(appearing as bright lines in a two dimensional image) forming an approximately $45^{\circ}$ angle to the horizontal. This channel continues to shrink in thickness and increase in length as the ambient fluid is pulled into the vortex.

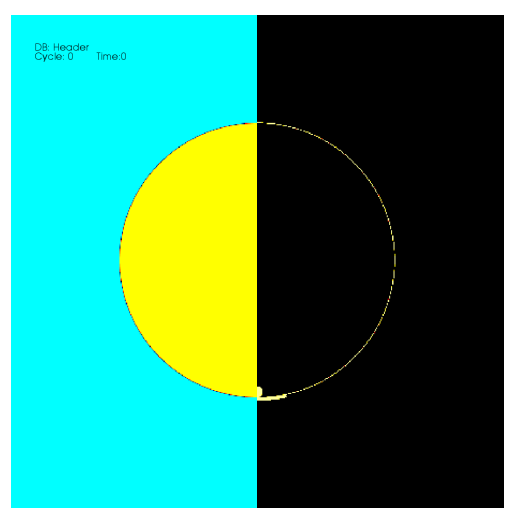

$(a)$

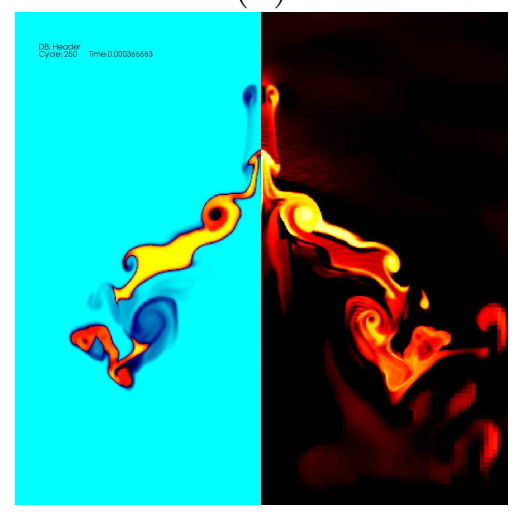

$(c)$

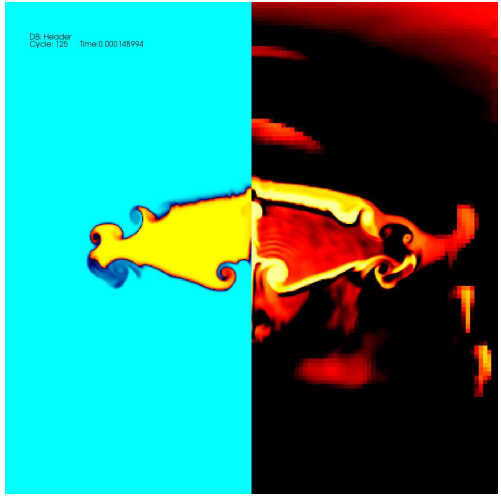

$(b)$

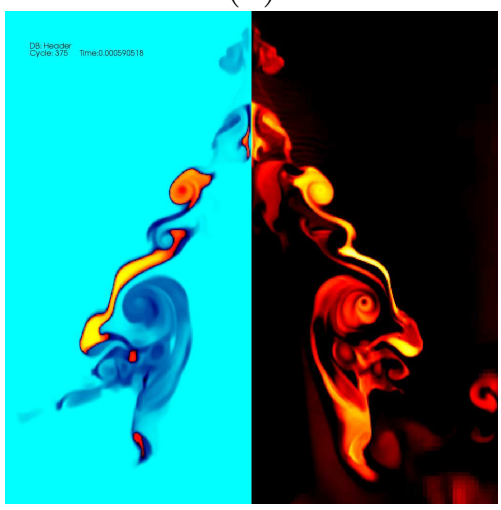

$(d)$

Figure 6: Argon volume fraction (left) and total density (right) fields from Raptor simulation of the interaction of a $M=2.88$ shock wave with a soap bubble filled with argon in a nitrogen environment. The soap film layer along with an exterior gravitational meniscus and interior droplet are included in the initial condition. Dimensionless times: $(a) \tau=0,(b) \tau=4.71,(c) \tau=13.33,(d) \tau=22.20$. Shock motion is from top to bottom.

The shock-bubble interactionfor an argon bubble enclosed in a soap film in a nitrogen environment is simulated using the 3D Eulerian adaptive mesh refinement (AMR) code Raptor. This code was developed at Lawrence Livermore and Lawrence Berkeley National Laboratories and has been used extensively in shock hydrodynamics calculations. The code integrates the 3D Euler (inviscid NavierStokes) equations forward in time using a scheme based on Godunov's method for shock capturing $[6,7]$. The AMR framework in which the integrator is embedded allows the solution to be resolved coarsely in smooth regions of the flow, thus minimizing computational cost $[8,9]$.

Results from these 3D simulations are shown in Fig. 6. The argon volume fraction (left) and total density fields (right) are plotted, showing, effectively, a slice through the datasets. A number of features are noticeable in these plots, which are also observed in the experimental images, including the formation of co-rotating primary and secondary vortex rings, the development of a strong, upward-directed axial jet, and a complex pattern of mixing at late times. Based on these simulations, 
it is clear that the presence of additional film material at the bottom of the bubble in its pre-shock state can lead to drastic effects in the post-shock flow. Simulations without film material have shown that shock diffraction, combined with a reflected rarefaction, can lead to the development of weak secondary upstream jet and vortex ring, but this effect become much more intense in the presence of film material, particularly if some of this material is concentrated near the bottom of the bubble. This is seen clearly in Fig. 6(b-d). Rather than a flow field dominated by a single vortex ring, we observe a complex cascade of vortical features in both simulations and experiments.

Further, in both simulations and experiments, we observe the breaking of axial symmetry in the shock-bubble interaction, particularly at late times. The experimental bubble has inherent asymmetry in its initial condition due to perturbations imposed by the bubble inflation and release processes; the simulated bubble has grid-seeded perturbations. At late times, these lead to the growth of azimuthal variations in the flow field, by a Widnall-type bending mode instability [5]. Vortex rings develop bends and kinks, and vortical fluctuations grow, leading to chaotic, turbulent motion in some regions of the flow. This is illustrated clearly in a 3D rendering of the late-time flowfield for the $M=2.88$ shock-bubble interactionfor an argon bubble in nitrogen, shown in Fig. 7 .

To characterize the growth of the mixed region resulting from the shock-bubble interaction, the maximum axial dimension ("height") and transverse dimension ("width") of the shocked bubble is measured both from experiments and from the Raptor simulations. These are plotted on the dimensionless timescale $\tau$ in Fig. 8, showing the Mach scaling of the mixing region growth. The experimental and numerical trends agree for the axial scale, but differences can be observed in the transverse scale, due to the difference in the densities of the experimental and numerically-modeled film material. The modeled film material has lower density, and shocks reflected from the bubble film and subsequently from the side walls are substantially weaker.

When the axial dimension of the bubble from lower-Mach-number experiments and simulations are compared to these data, a significant deviation is observed. That is, the timescaling based on $W_{t}$ fails at lower Mach numbers, and the growth trends, shown in Fig. 9, are much slower on the dimensionless timescale for $M=1.33$ than for higher Mach numbers. It is postulated that this effect is due to the transition, as the incident Mach number increases beyond $M=2.07$ in nitrogen, to post-shock flow velocity $u_{1}^{\prime}$ that is supersonic in the fixed reference frame $\left(u_{1}^{\prime}>c_{1}^{\prime}\right)$.

\subsubsection{Divergent geometry, high density contrast}

Figure 10 shows the evolution of the shocked helium bubble after contact with a $M=2.95$ shock wave with a velocity of $1040 \mathrm{~m} / \mathrm{s}$ in the nitrogen. The particle velocity behind the shock in the nitrogen is $u_{1}^{\prime}=767 \mathrm{~m} / \mathrm{s}$ and the transmitted wave velocity in the helium is $W_{t}=1971 \mathrm{~m} / \mathrm{s}$. The initial stage of the shock interaction with the bubble results in the compression of the bubble as seen in Fig. 10(a), where the transmitted shock wave is still inside the bubble. The top bright portion of the image is the upstream part of the bubble that has been shocked and the lower horizontal bound of this region is the shock front. During this period of shock interaction, vorticity is deposited due to the misalignment between the pressure and density gradients which later leads into the formation of the PVR. Figure 10(b) shows the bubble after the shock wave has completely traversed it. It can be seen that the top portion of the bubble is caving inwards, on axis, due to the vorticity deposited during the interaction phase. Figure $10(b)$ shows a good qualitative comparison with the image 


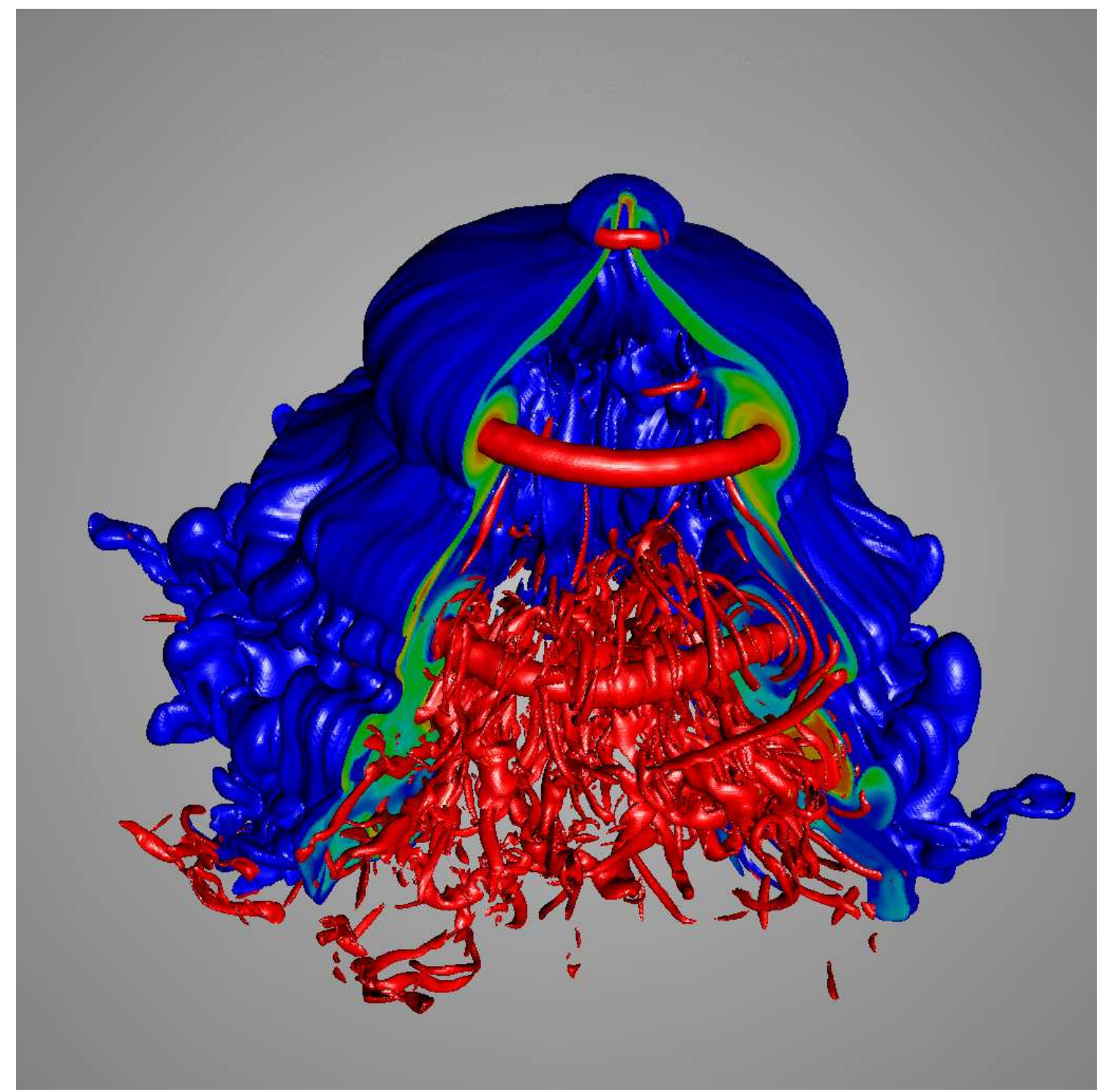

Figure 7: Argon bubble with soap film, shocked at $M=2.88$ in a nitrogen environment, from a 3D simulation using Raptor. The 0.1 isosurface of argon volume fraction is shown in blue, and an isosurface of vorticity magnitude is shown in red in the cut-out region. The bubble is shown at a dimensionless time $\tau=20.14$. 

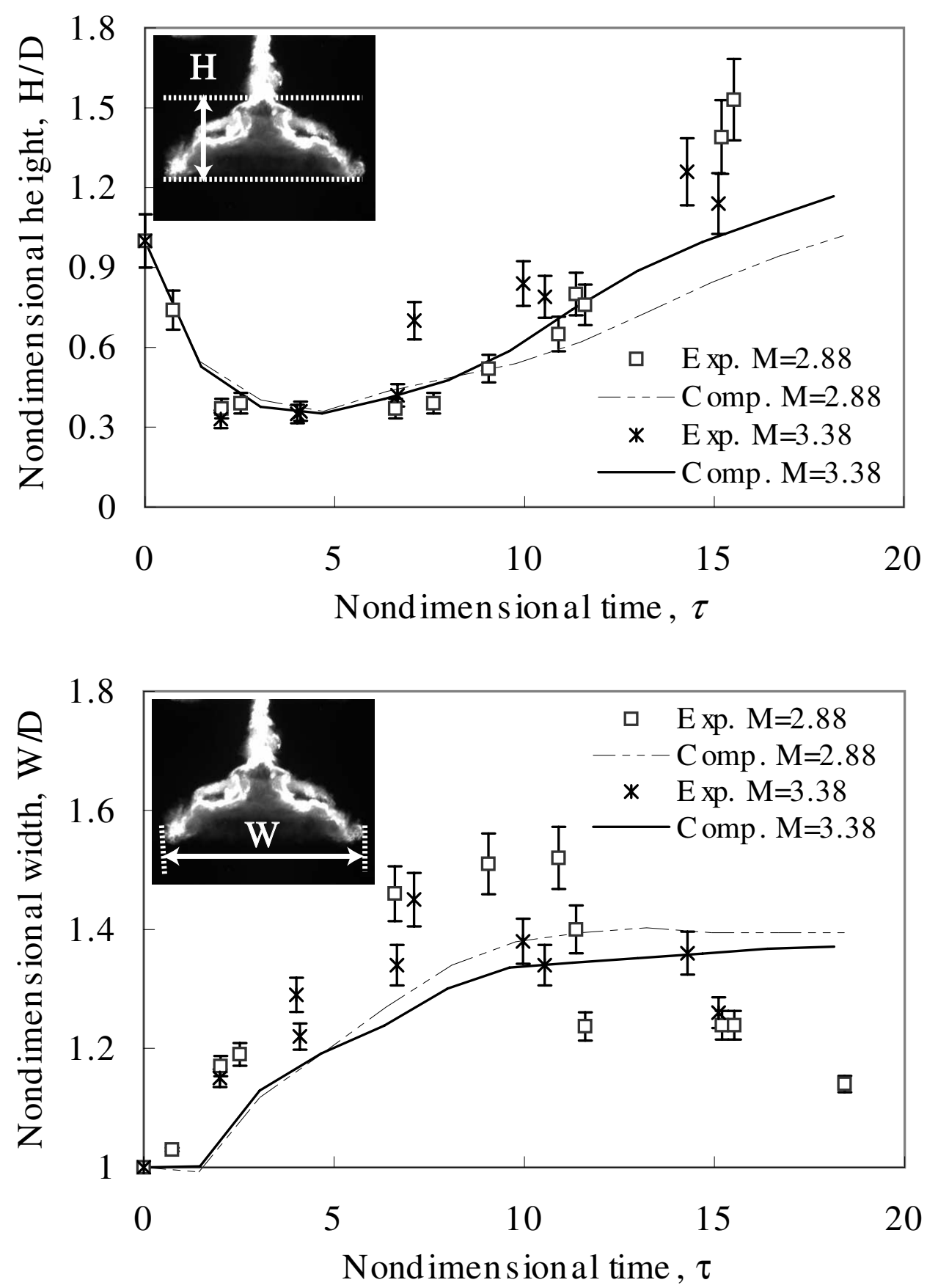

Figure 8: Non-dimensional length scale of an argon bubble accelerated by $M=2.88$ and 3.38 shock as functions of non-dimensional time. Upper: transverse scale ("width"); lower: axial scale ("height").

(Fig. 1, frame 2) from the Layes et al. experiment [10] which was conducted at a low Mach number. This caving-in is analogous to the phase reversal observed in single-mode H/L RMI experiments. The observed caving-in is a manifestation of $\nabla \rho \times \nabla p$ vorticity deposition. 


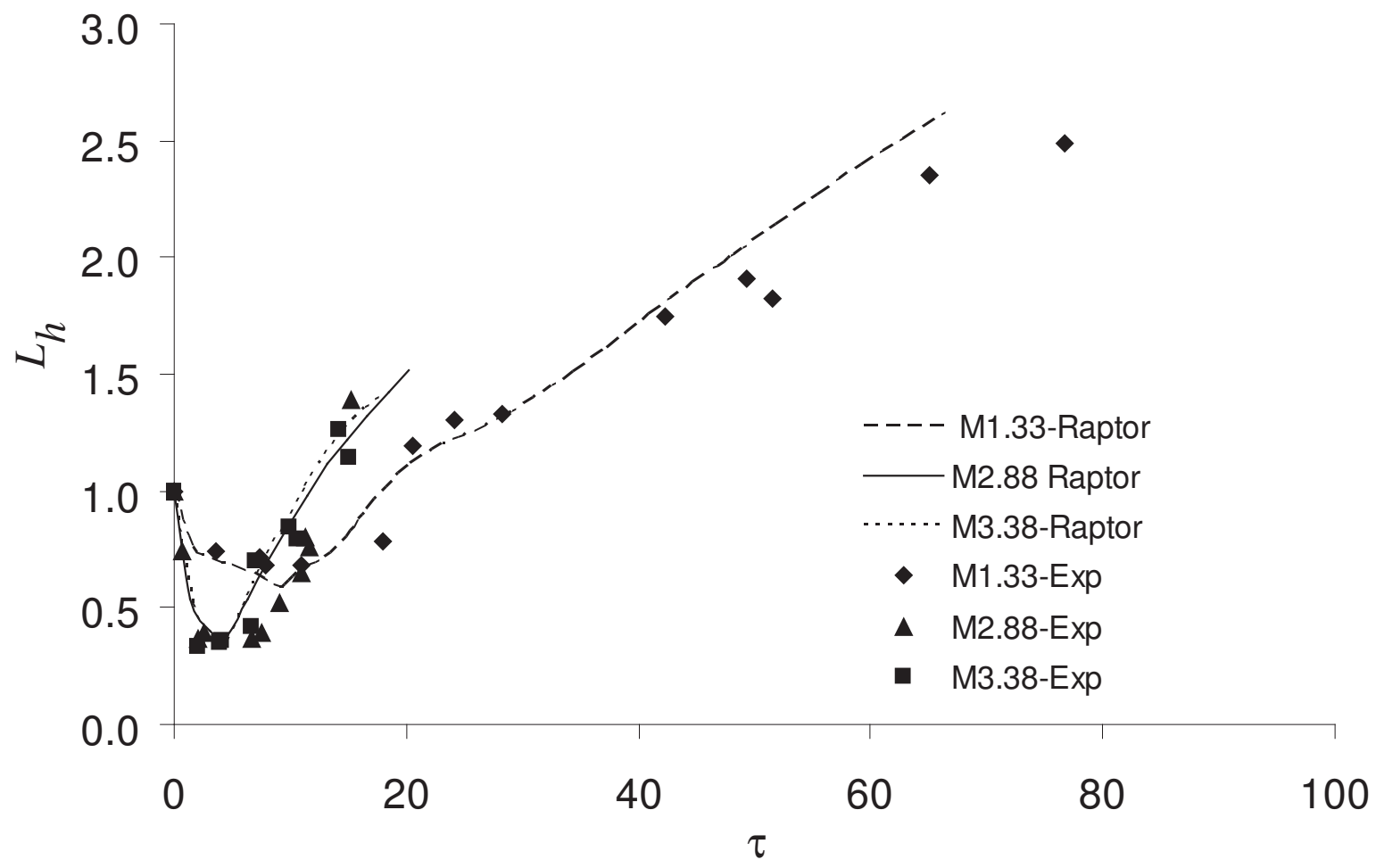

Figure 9: Non-dimensional length scale of an argon bubble accelerated by $M=1.33,2.88$ and 3.38 shock as functions of non-dimensional time.

In Fig. 10(c) the formation of the PVR is observed. The white loops on the left and right of the image, with interior dark spots, are the cross section of the vortex ring at its mid-plane. The visualization technique highlights the fringes of the vortical cores but leaves the core itself as a distinct dark spot due to light-scattering soap film particles being centrifuged outwards. That is, the relatively dense soap film particles are displaced from the vortex core by centrifugal forces that arise due to the large angular momentum of the vortex.

Associated with this centrifugal effect on entrained liquid particles, a radial-outward pressure gradient arises in the vortex, which traps helium in the core, thus inhibiting helium-nitrogen mixing. Therefore, we conclude that the dark core seen in the PVR consists primarily of helium which is pulled inside the vortex ring from the center of the bubble without having mixed with the nitrogen. The next image in time, Fig. 10(d), has three notable features, the first being that the PVR entrains the fluid from the center portion of the bubble and swirls it into the vortex ring. If one looks at the right side of the image, the sense of rotation in the vortex ring is counterclockwise. The second feature is the disk-shaped head of the bottom of the bubble, which appears as a bright horizontal swath in the 2D image connecting the left and right loops. The third feature is an annulus (it appears as a pair of bright vertical arcs in the 2D image) connected to the vortex ring and the top of the bubble. The orientation of these arcing filaments indicates the motion of helium being swept from the bubble interior into the PVR. Figure $10(d)$ and $10(e)$ show good agreement with each other for images obtained from two different experiments. Figure $10(f)$ shows that the PVR has entrained almost all the fluid from the center of the bubble. The size of the ring has grown considerably compared to Fig. 10(c). To summarize this early phase of the interaction, the bubble 


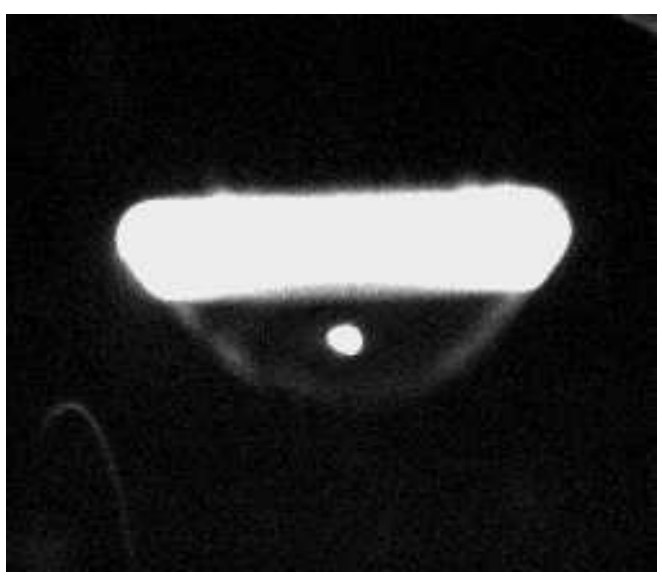

$(a)$

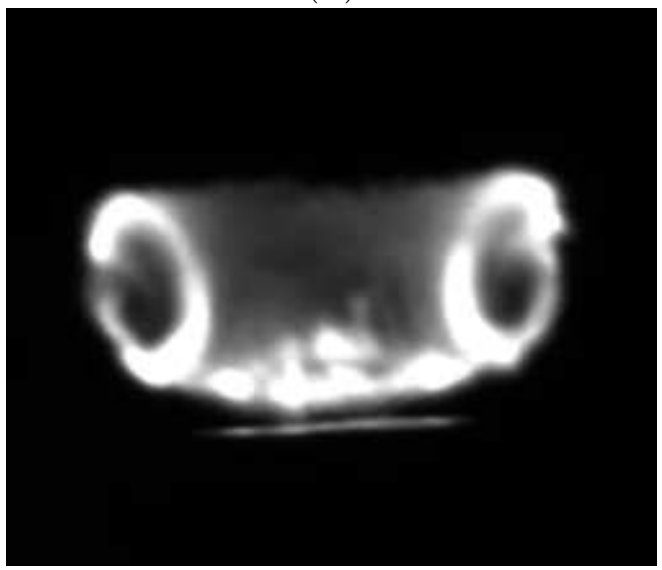

$(c)$

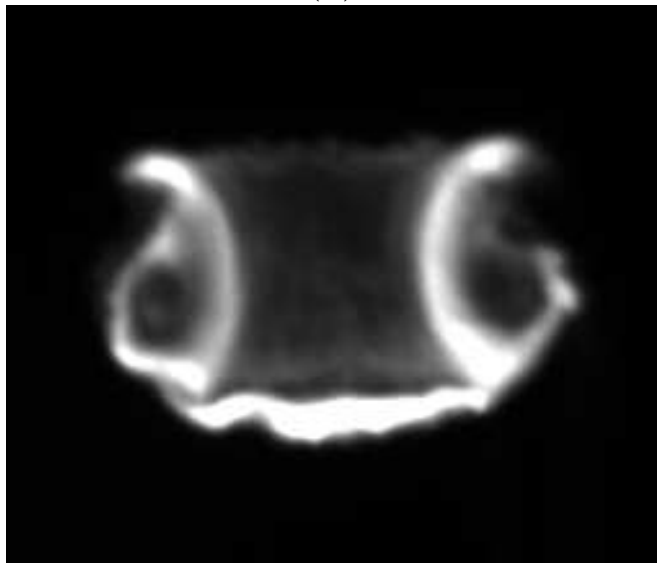

(e)

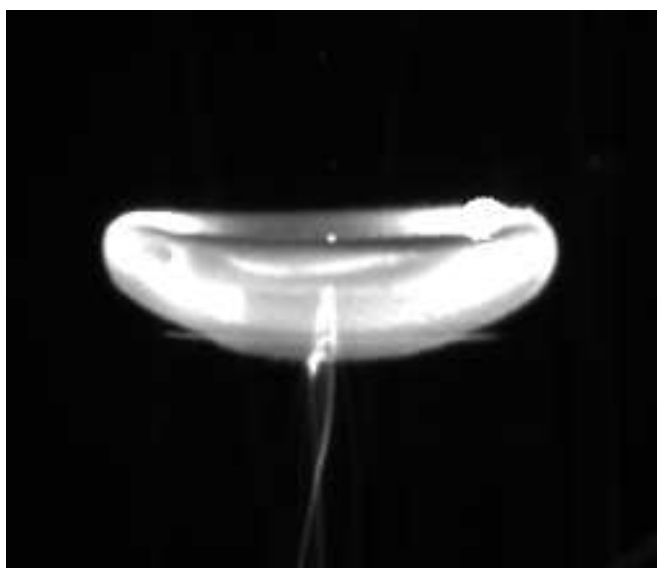

$(b)$

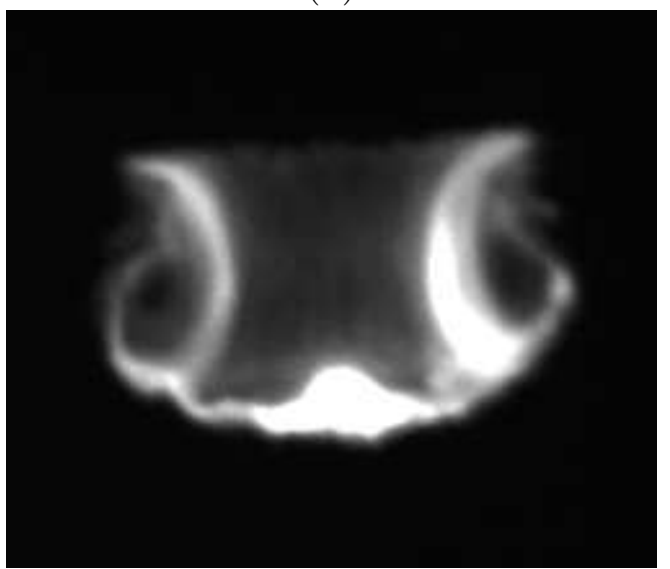

$(d)$

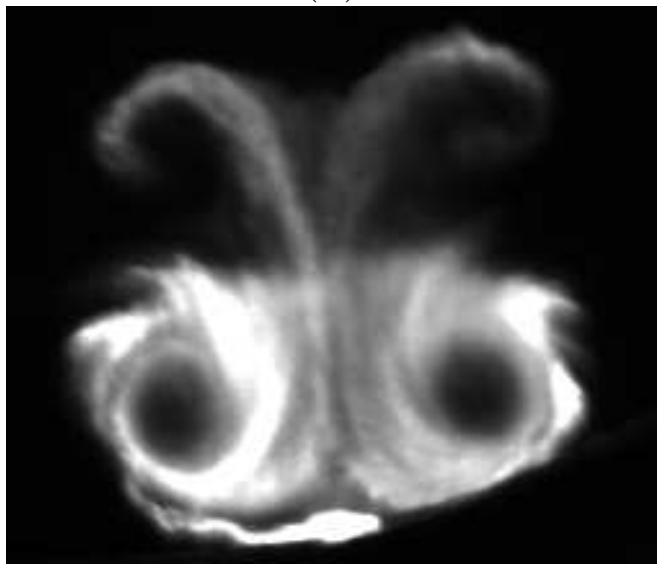

$(f)$

Figure 10: Experimental images of a shocked $(M=2.95)$ helium bubble in the nitrogen: $(a) \tau=1.3$, (b) $\tau=4.0,(c) \tau=7.7,(d) \tau=11.4,(e) \tau=11.6$, and $(f) \tau=23.8$. The shock wave travels from the top of the image to the bottom.

dynamics are dominated by the formation and growth of the PVR which leads to the mass transfer from the center of the bubble to the PVR. This can also be seen in the experimental measurements of Layes et al [11]. The curving of the upstream part of the annulus shows the start of the secondary 
features in the flow.

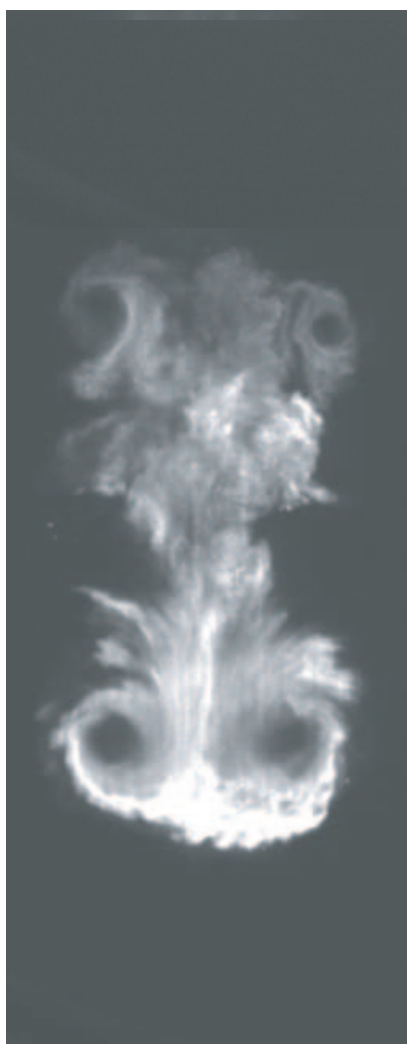

$(a)$

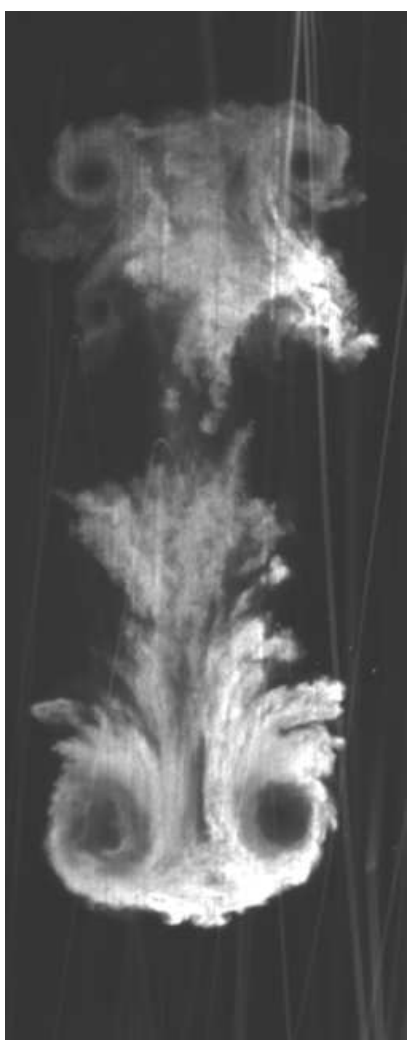

(b)

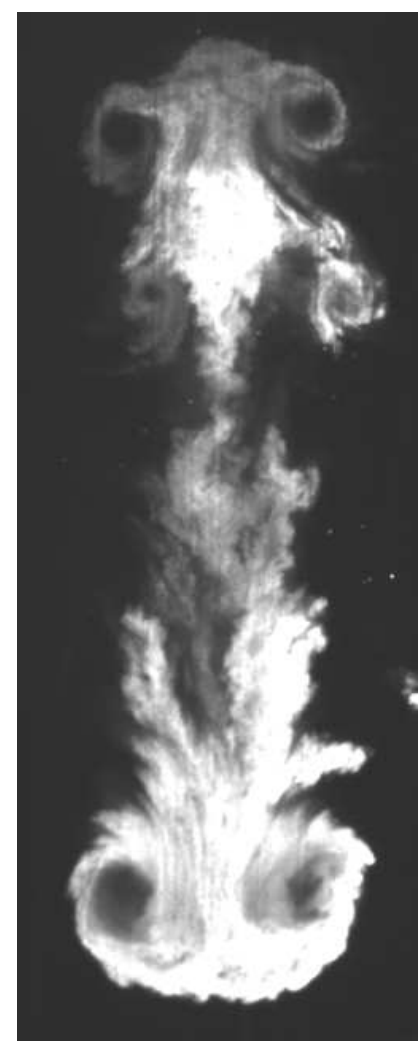

$(c)$

Figure 11: Experimental images of a shocked $(M=2.95)$ helium bubble in the nitrogen showing secondary and tertiary vortex rings: $(a) \tau=46.3$, (b) $\tau=63.5$, and $(c) \tau=69.5$.

Figure 11 shows the presence of secondary and tertiary counter-rotating vortex rings in the flow field. These features are absent in the case of low Mach number experiments $[1,10]$. These features are generated by a secondary baroclinic source of vorticity associated with the slip line traced in the flow by the Mach stem and triple point which develop at the early stages of the shock-bubble interaction, due to irregular shock refraction. This was deduced from the simulations carried out for the given experimental scenario [12]. Helium is stripped off from the top part of the PVR in low concentration, and is then entrained by the SVR.

In Fig. 11( $a$ ) some fine-scale turbulent structures can be seen on the bottom of the PVR. In Fig. $11(b)$ and $11(c)$ one can clearly identify the three distinct vortex rings: the PVR at the bottom of the image, the SVR at the top of the image and a tertiary vortex ring (hereafter referred to as TVR) just below the SVR. The SVR and TVR have the same sense of rotation (opposite to the PVR) due to which the region between them has an alternating vorticity field associated with a stagnation-type flow field [12]. Comparing the sense of rotation in the SVR formed in the H/L case to that for the $\mathrm{L} / \mathrm{H}$ case [3], it can be concluded that the rotation in the SVR is independent of the bubble gas (clockwise rotation in both cases) even though the formation mechanism is different. It can be surmised from these images that the strongest vorticity field is associated with the PVR. Most of the fluid (ambient nitrogen) in the region between the TVR and PVR is being pulled towards the PVR. This is observed from the long tails, or dendrite-like structures, associated with 
the PVR, visible in Fig. 11(b). One can also observe that the core of the PVR becomes distorted and no longer has a circular cross-section. Finally, in Fig. 11(b) and 11(c), the pinching-off of the SVR and TVR from the PVR can be seen.

Several relevant length scales are visible in the vortex dynamics of the shock-bubble interaction as shown in Fig. 12. The largest is the axial distance between the primary and the tertiary vortex ring (scale 1). The second scale (scale 2) can be associated with the geometrical features of the primary vortex ring formed due to the initial baroclinic vorticity deposition. The smallest scale is that of the features associated with secondary instabilities like the Kelvin-Helmholtz instability seen on the edges of the PVR.

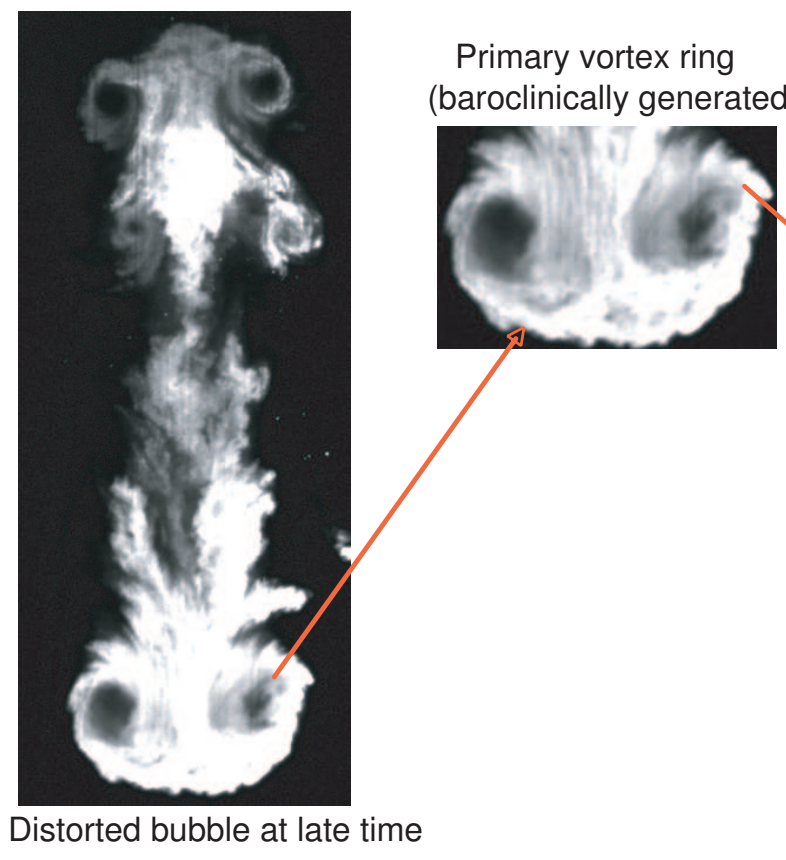

Scale 1
Scale 2

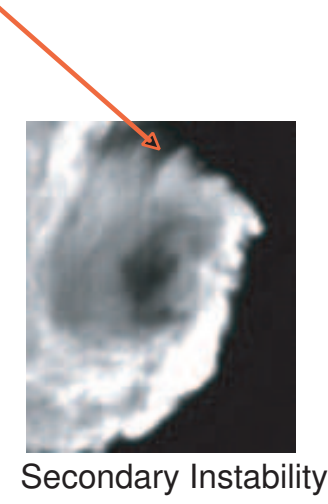

Scale 3

Figure 12: Length scales observed in shock-bubble interaction.

Simulations for the divergent scenario of the shock-bubble interactionare also carried out using the Eulerian AMR code Raptor. These are set up on a 3D Cartesian mesh, with a planar shock wave of specified strength incident on a helium bubble in a nitrogen environment. Results from these simulations are shown in Fig. 13.

Divergent shock refraction can be seen in Fig. 13(a), followed by the formation of a vortex ring whose sense of rotation is opposite to that formed in the convergent scenario discussed above. Mixing of the bubble fluid into the ambient fluid is suppressed in the shocked helium bubble, because the density gradient associated with the centrifugal effect in the vortex core tends to trap the lighter helium fluid in the vortex, isolating it from the ambient fluid. The formation of a secondary, counter-rotating vortex ring is seen in Fig. 13(e-f). A slip line is traced in the flow by the Mach stem connecting the incident and transmitted shocks. This slip line rolls up and forms the vortex ring shown in Fig. 13(f), which has opposite-signed vorticity relative to the primary vortex ring.

The late-time flow field shows the significant axial elongation undergone by the bubble in the post- 


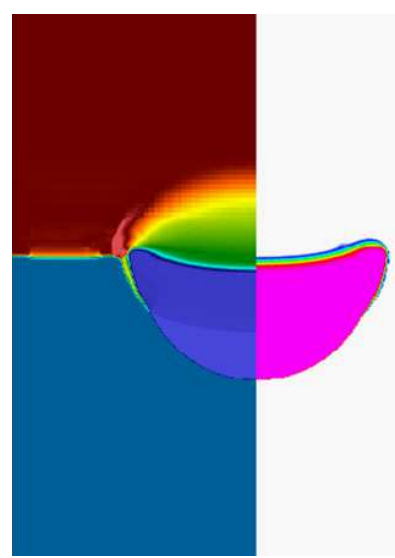

(a)

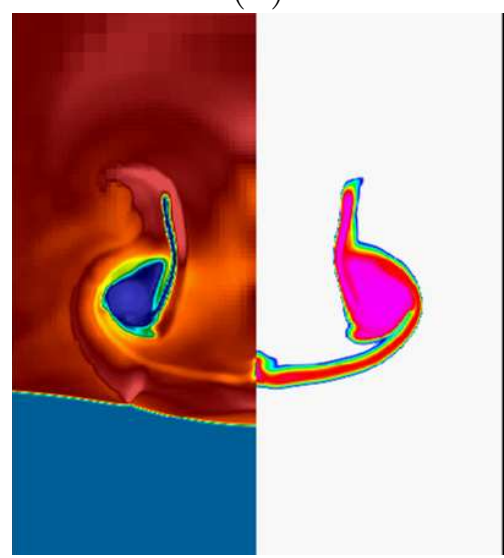

(c)

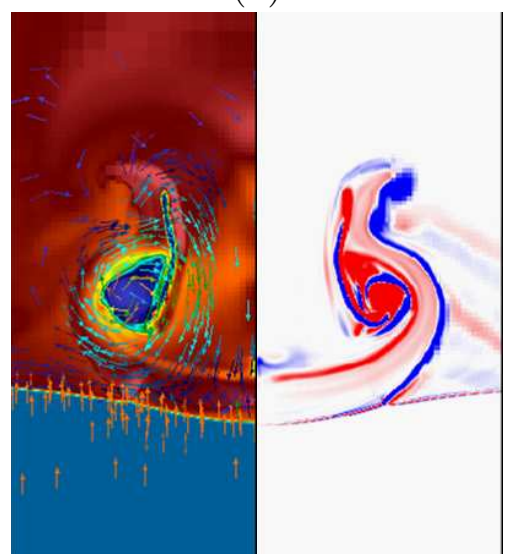

$(e)$

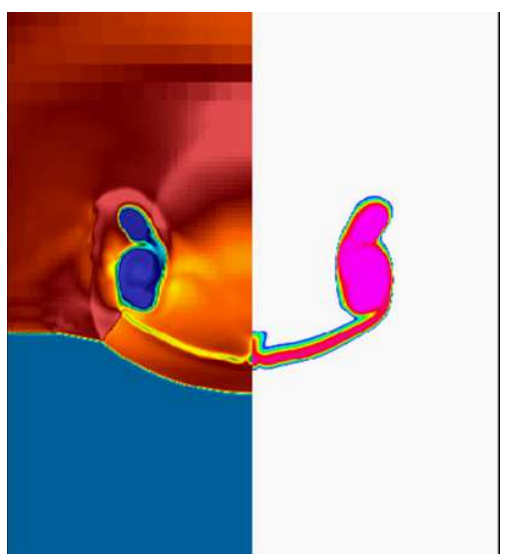

$(b)$

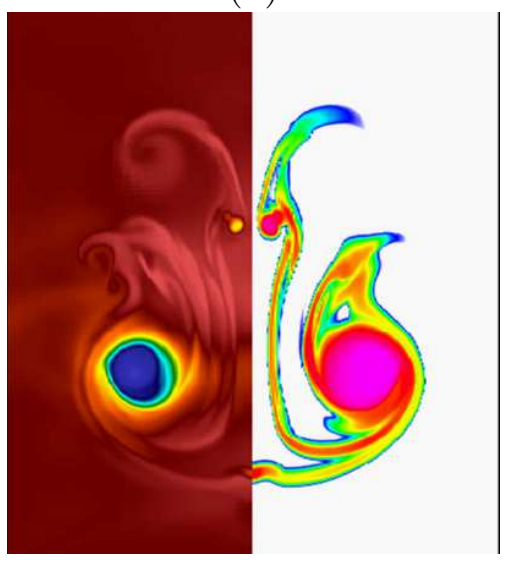

$(d)$

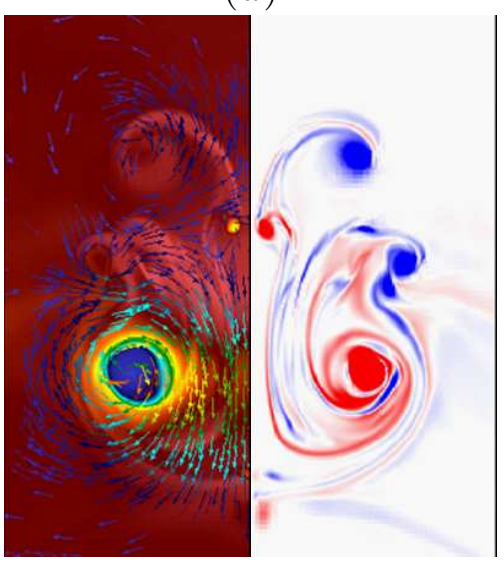

$(f)$

Figure 13: Total density (left) and helium volume fraction (right, log scale) fields from Raptor simulation of the interaction of a $M=2.95$ shock wave with a helium bubble in a nitrogen environment (soap film excluded). Dimensionless times: $(a) \tau=1.3,(b) \tau=7.7,(c) \tau=11.5,(d) \tau=23.6,(e)$ $\tau=11.5,(f) \tau=23.6$. Shock motion is from top to bottom. Density (left) and vorticity (right) fields, with velocity vectors overlaid, are shown in $(e)$ and $(f)$.

shock flow, as well as the further development of the secondary vortex ring, and an additional "tertiary" vortex ring, also rotating in the opposite sense to the primary vortex ring. These are 
shown in Fig. 14.

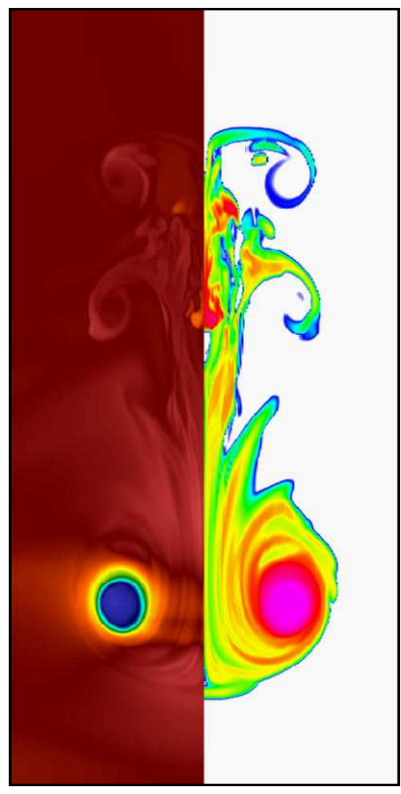

$(a)$

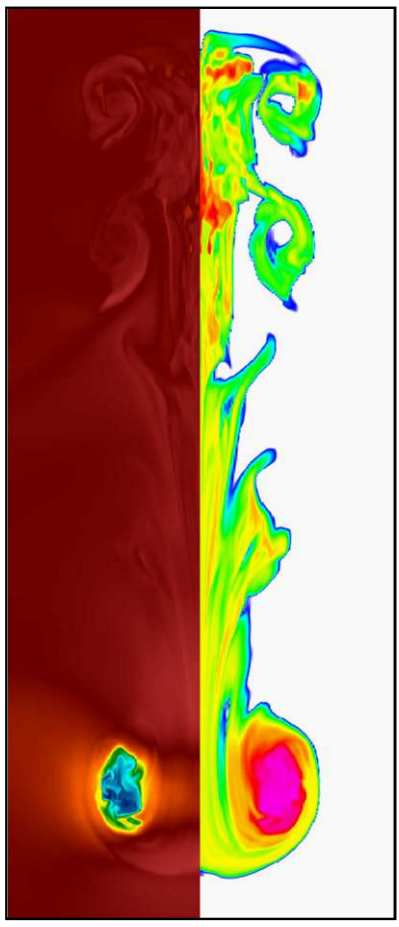

$(b)$

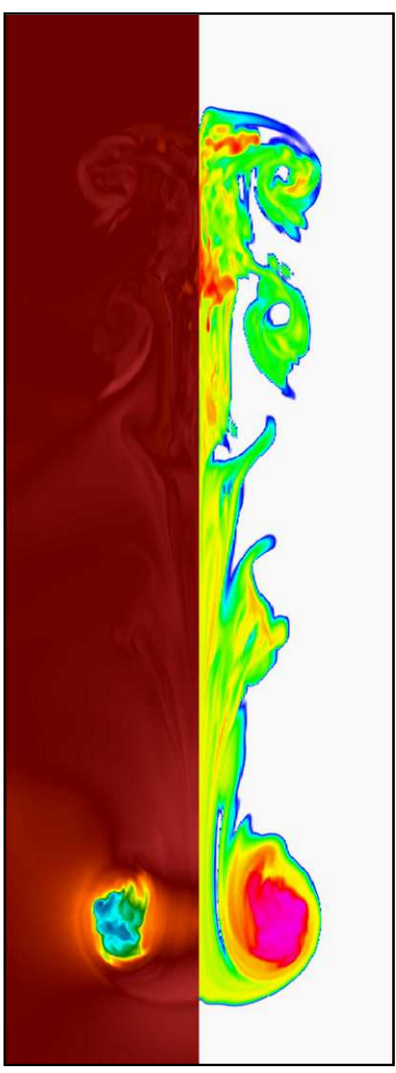

(c)

Figure 14: Total density (left) and helium volume fraction (right, log scale) fields from Raptor simulation of the interaction of a $M=2.95$ shock wave with a helium bubble in a nitrogen environment (soap film excluded). Dimensionless times: $(a) \tau=46.3,(b) \tau=63.5,(c) \tau=71.6$. Shock motion is from top to bottom. Density (left) and vorticity (right) fields, with velocity vectors overlaid, are shown in $(e)$ and $(f)$.

The axial and transverse growth trends of the shocked helium bubble for 2.95 are shown in Fig. 15.

The axial growth trends of the shocked helium bubble for $M=1.4, M=2.08$, and $M=2.95$ are all shown together in comparison with the corresponding trends obtained from Raptor simulations in Fig. 16.

\subsubsection{Computational parameter study}

The time-dependent integral properties of the shock-bubble interactionare also examined using a series of 3D simulations with Raptor designed to span the parameter space of previous work on this problem, within the regimes accessible to mechanical shock tubes. Twelve scenarios are considered: three shock strengths each for air-helium, nitrogen-argon, air-krypton, and air-R12. The average density of the bubble fluid over time is computed from the simulations for each scenario and compared to the model of Giordano and Burtschell (2006) [13], based on 1D gasdynamics. The average density is recast as the normalized compression $\tilde{C}(t)$, using 


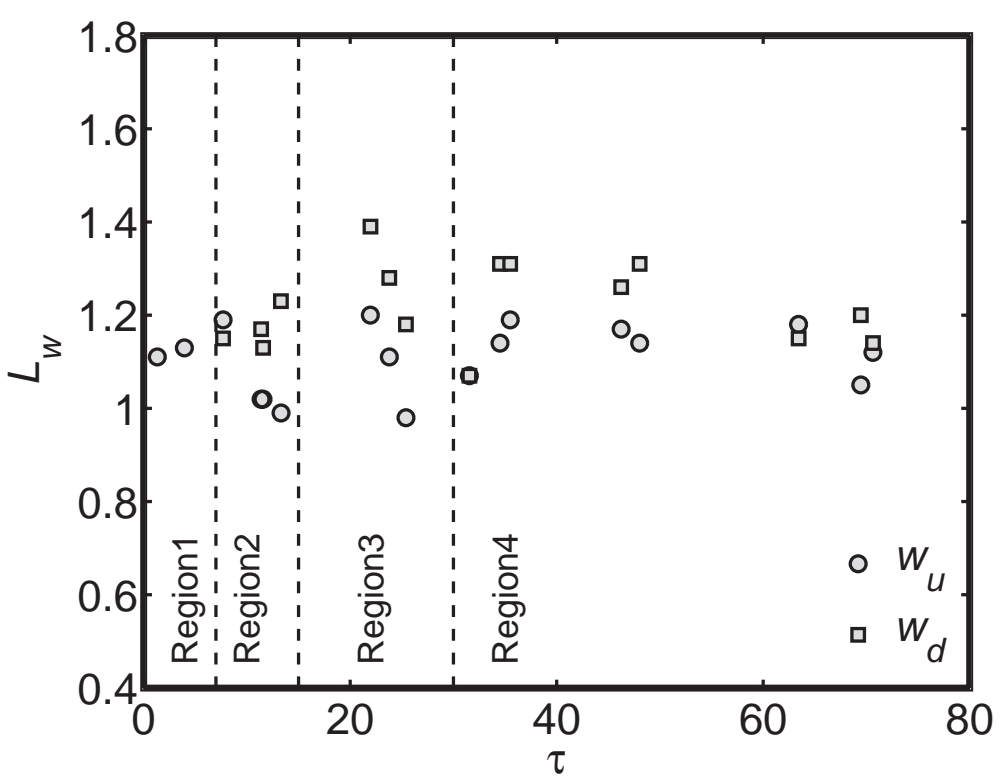

(a)

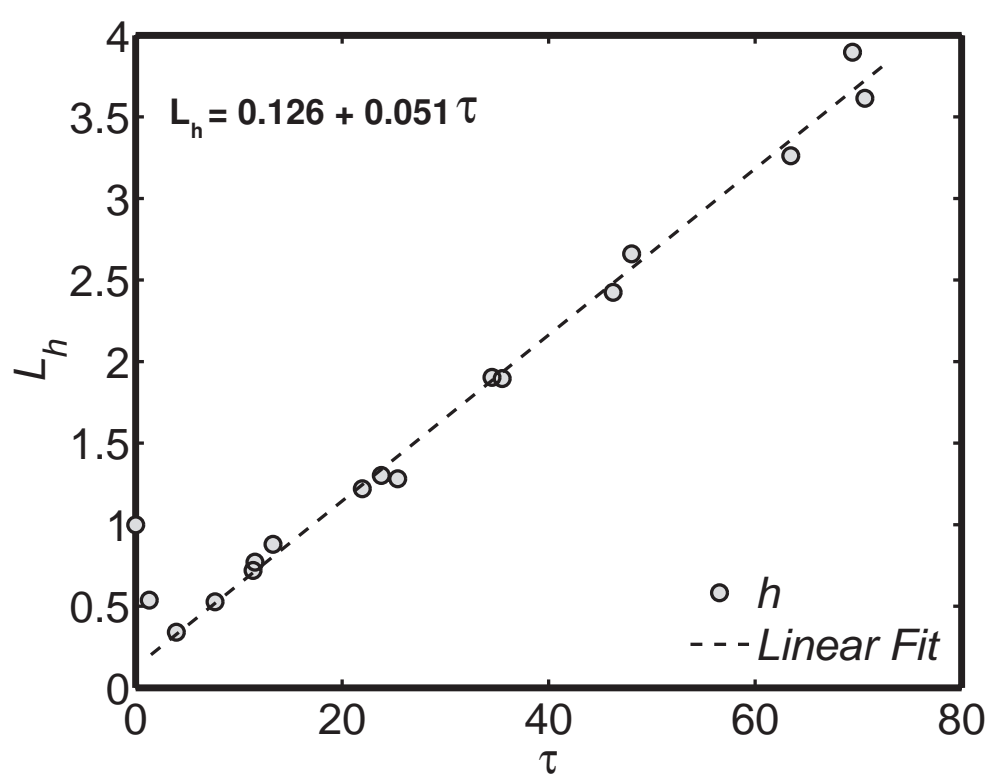

(b)

Figure 15: Non-dimensional length scales of helium bubble accelerated by $M=2.95$ shock as functions of non-dimensional time: $(a)$ width, and $(b)$ height.

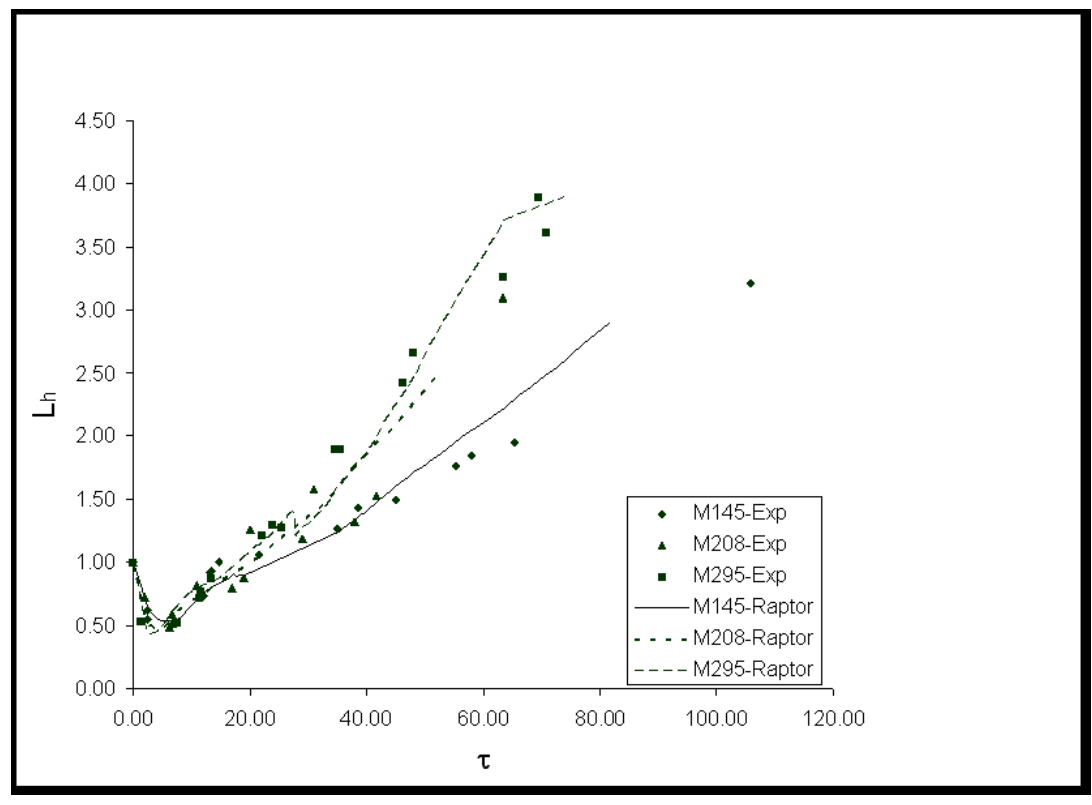

Figure 16: Axial dimension ("height") of shocked helium bubble at $M=1.4,2.08$, and 2.95, from experiments and simulations.

$$
\tilde{C}(t)=\frac{\langle\rho(t)\rangle-\rho_{2}}{\rho_{f}^{1 D}-\rho_{2}},
$$


where $\langle\rho(t)\rangle$ represents the volume-averaged partial density of bubble fluid at time $t$ from simulations, $\rho_{2}$ is the initial (unshocked) bubble fluid density, and $\rho_{f}^{1 D}$ is the density predicted by a 1D gasdynamics analysis [13]. The trends in normalized bubble compression are shown in Fig. 17.
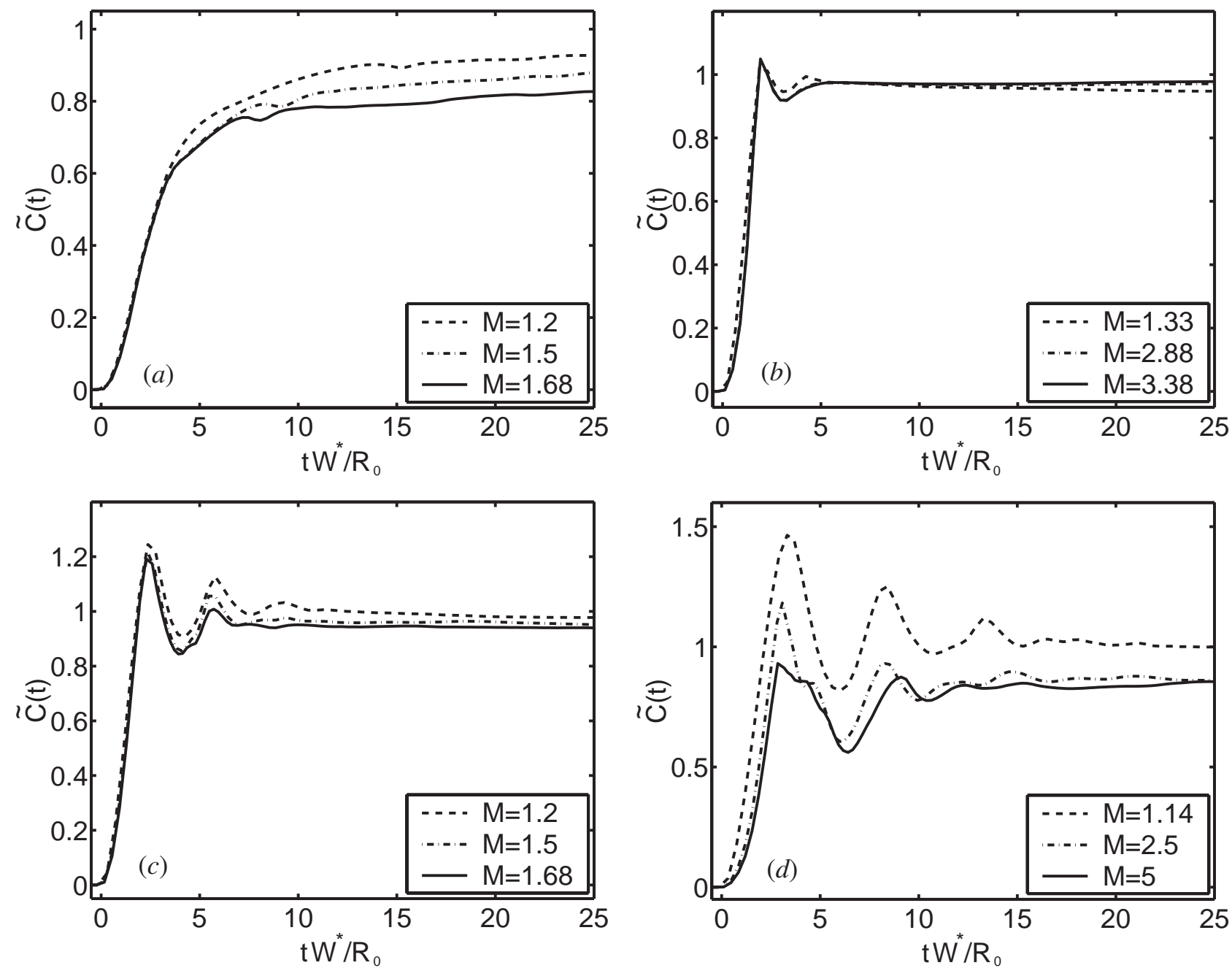

Figure 17: Plots over dimensionless time of the shocked-bubble compression factor obtained from three dimensional simulations, normalized to the compression factor obtained from the onedimensional gasdynamics model of [13], using (1): (a) Air-He, (b) $\mathrm{N}_{2}$-Ar, (c) Air-Kr, and (d) Air-R12.

The trends in Fig. 17 show that the compression history of the bubble collapses nearly to a single trend, under this normalization and timescaling, within each gas pairing. However, the patterns of scattered shocks and rarefactions generated in the shock-bubble interactionvary significantly across different gas pairings. These waves are trapped in the bubble in the convergent cases (Fig. $17 b-d)$, resulting in oscillations about the 1D gasdynamics prediction, which persist later in time as the density contrast increases. In the divergent scenarios (Fig. 17a), however, the waves are refracted away from the bubble, so that no oscillations are observed, and the bubble never reaches the compression predicted by $1 \mathrm{D}$ gasdynamics.

Thus, significant differences are observed between features observed in the shock-bubble interaction- 
for different gas pairings, and scaling can only be achieved successfully for fixed Atwood number. This is primarily due to the strong pattern of secondary scattered shocks and rarefactions arising in the shock-bubble interactionwhen the density contrast is large. These secondary waves interact with the deformed bubble and induce the development of a highly complex pattern of vortical perturbations at late time, leading to a chaotic, highly-mixed flow field at late times. This is illustrated in Fig. 18, which shows the late-time flow field for the air-R12 $(A=0.613)$ scenarios at shock strengths $M=2.5,5.0$.

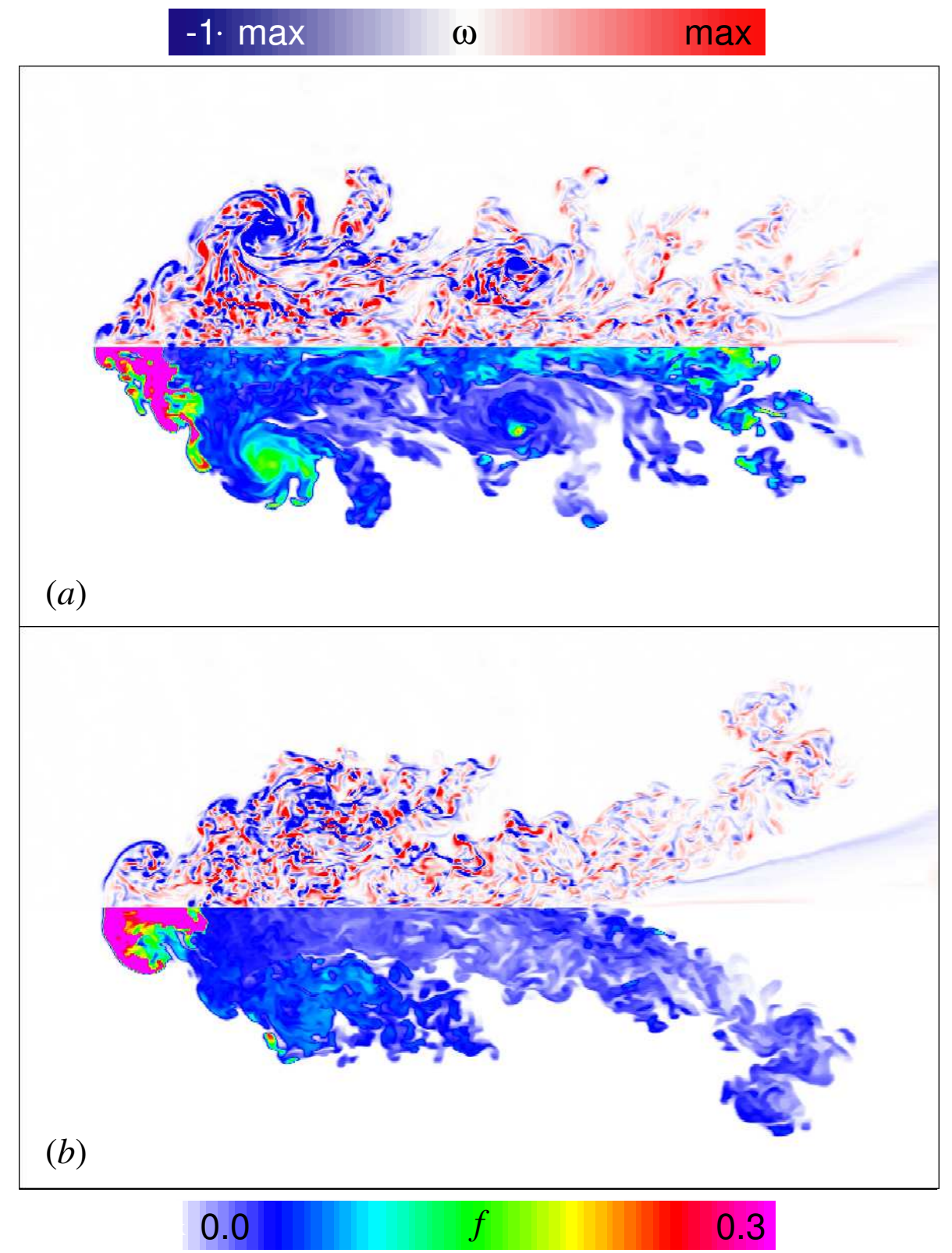

Figure 18: Vorticity magnitude (upper) and R12 volume fraction (lower) fields for the air-R12 scenario, at $t=25 R / W_{i}$, illustrating the turbulence-like flowfield generated in the shock-bubble interaction at late times. (a) $M=2.5,(b) M=5.0$. 


\subsection{Impulsively accelerated 2-D interfaces}

\subsubsection{Background}

The literature of previous investigations of impulsively-accelerated 2-D interfaces is very vast, starting with the pioneering work of Meshkov [14]. The earliest work focused on the behavior of a single-mode, infinitely thin interface with small initial amplitude $(\eta<<\lambda$, where $\eta$ and $\lambda$ are the perturbation amplitue and wavelength, respectively) during the early post-shock stage (the "linear" stage) and showed that, during that phase, the perturbation grows linearly in time.

The objects of the currently ongoing investigations worldwide are: i) the late, non-linear stages, when the amplitude is no longer small compared to the wavelength; ii) modal interactions for non single-mode interfaces; iii) 3-D effects; iv) the transition from an interface to a mixing layer.

\subsubsection{Experimental setup}

A technique analogous to that first used by Jeff Jacobs $[15,16]$ at the University of Arizona has been developed to prepare a 2-D sinusoidal interface without resorting to a membrane or a retractable plate. A schematic of the system is shown in Fig. 19: two different gases flow into the shock tube from above and below the desired interface location and meet head-on forming a stagnation flow that generates a flat interface; two pistons, in opposite shock tube walls, extending about $3 \mathrm{~cm}$ both above and below the plane of the stagnation flow, oscillate syncronously extending out of and retracting into the walls, imposing a standing wave onto the interface whose modal content can be adjusted by varying the amplitude and frequency of the pistons' oscillations. Schematics of the tube assembly and of the oscillating pistons system are shown in Fig. 19 while an image of the actual pistons installed in the interface section is shown in Fig. 20.

The interface is visualized once before and twice after interaction with the shock wave. Both the PLIF and PMS techniques present advantages and challenges, Mie scattering being easier to implement but yielding no quantitative concentration data, PLIF requiring image normalization algorithms to account for laser sheet divergence, attenuation and refraction. A "mean" interface line is extracted from each planar view by selecting the location in each column of the image array where the pixel value is $50 \%$ of the difference between the maximum and minimum pixel values in that column. The shape of the pre-shock interface mean line can be analyzed with standard Fourier transform techniques to asses its spectral content.

Figure 21 shows the spectrum of a typical initial condition mean line produced by oscillating the pistons at a frequency of $2.6 \mathrm{~Hz}$ and an maximum displacement of $2.5 \mathrm{~cm}$ : mode 3 appears to be the dominant one; all other modes between 1 and 7 have small amplitudes compared to mode 3; modes 8 and up have negligible amplitude.

Detailed knowledge of the initial conditions is important for two reasons: the values of the initial amplitude and wavenumber are used to normalize the post-shock amplitude and times, as shown below; and the spectrum of the pre-shock mean line is used to initialize Raptor calculations aimed at reproducing the experimental results. Since one single calculation is supposed to reproduce the results from several experimental runs, the average spectrum from all the experimental runs is used in the initialization. Furthermore, different Raptor runs are performed using all or few of the first 100 modes in the spectrum: the best results, shown in Sec. ?? below, are obtained using only five 


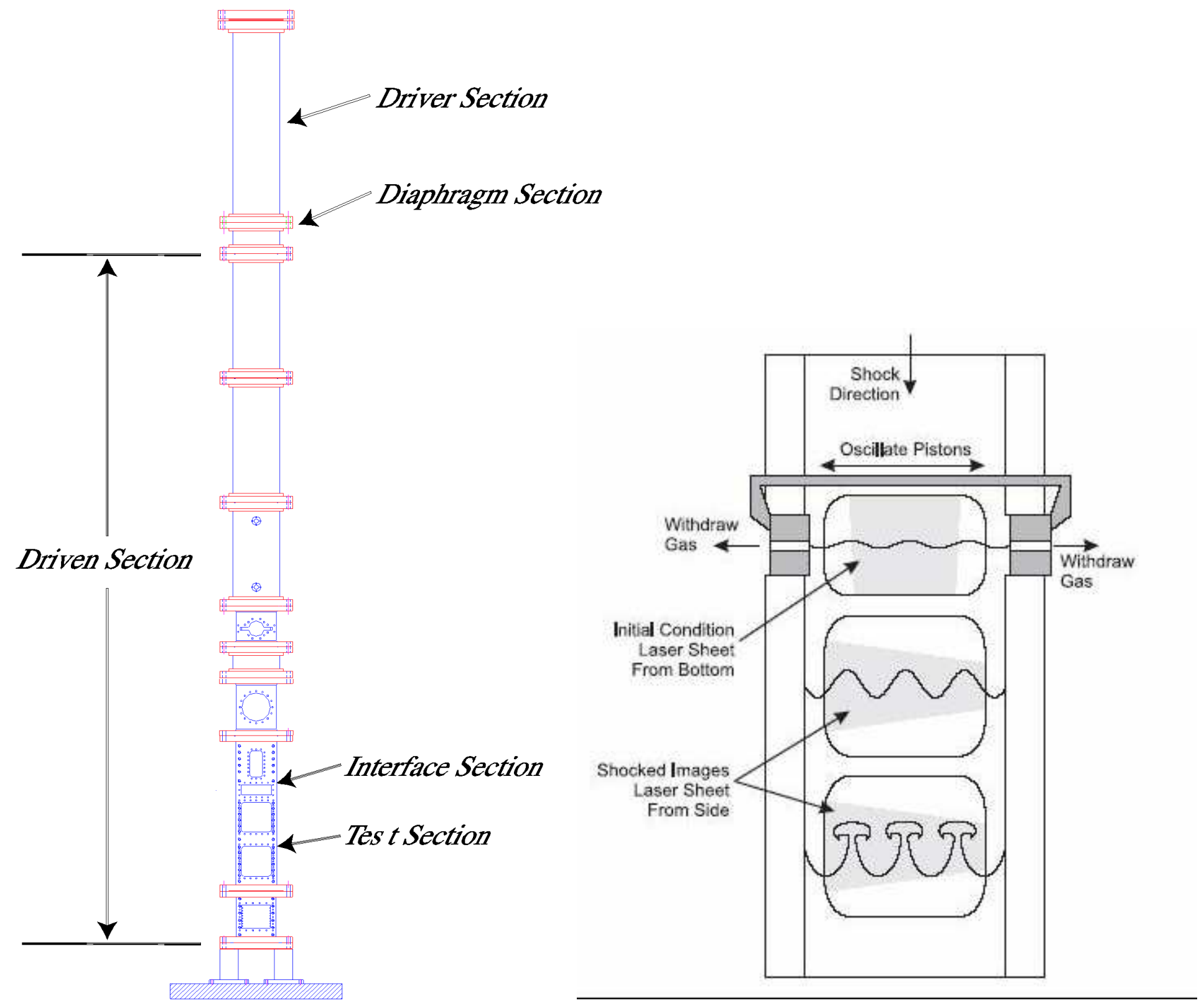

Figure 19: Schematics of the shock-tube facility and of the oscillating pistons system.

modes.

To ensure that the interface produced with the oscillating pistons technique is indeed 2-D, planar images of the unshocked interface were taken with the laser sheet in five different planes between the shock tube midplane and the shock tube back wall. An overall interface shape was then approximated using the mean lines from the five images and a 3-D reconstruction algorithm. The results (a sample is shown in Fig. 22) indicate that the interface is essentially 2-D in the central portion of the shock tube (where all the imaging takes place in the actual experiments) with 3-D effects limited to regions near the walls where the boundary layers are. 


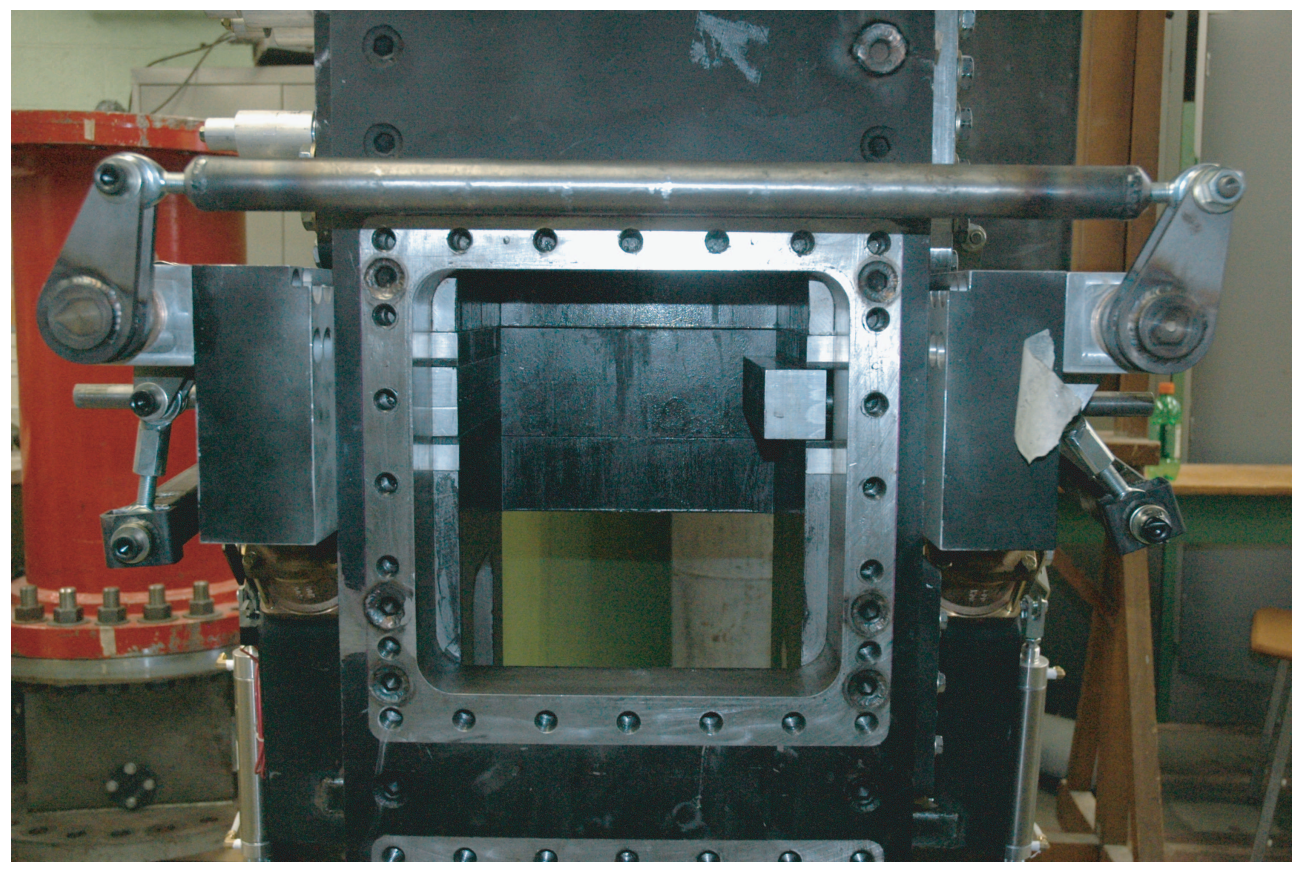

Figure 20: View of the oscillating pistons.

\subsubsection{Results}

Experiments have been performed using shock strengths of $1.3<M<2.05$, with $\mathrm{N}_{2}$ flowing downward from an injection port $1 \mathrm{~m}$ above the test section; and $\mathrm{SF}_{6}$ flowing upward from the tube end wall. This gas pair corresponds to an Atwood number $A=0.68$. In some cases, the $\mathrm{N}_{2}$ was doped with acetone ( $\sim 28 \%$ volume fraction, corresponding to saturation) to perform planar laser induced fluorescence (PLIF) while in others the dopant was cigarette smoke and imaging of the interface was based on planar Mie scattering (PMS).

Figure 23 shows sample images from from an $M=1.3$ experimental series with cigarette smoke as flow tracer and the corresponding results from a numerical experiment with the Raptor code initialized with the average FFT spectrum of the initial conditions of all experimental runs. Time is normalized with the wavenumber $k$ and the initial post-shock growth rate $\dot{\eta}_{0}$.

Sample images from an $M=2.05$ experimental series with the same gas pair are shown in Fig. 24.

A first qualitative result is that some of the same features (e.g. the development of narrow spikes and broad, nearly flat bubbles) appear in both sets of images, at earlier normalized times in the case of the $M=2.05$ series.

The amplitude of the mean line of the post-shock interfaces is used to measure the growth rate of the perturbations (initially a sinusoid; later spikes and bubbles). The amplitude is normalized with the wavenumber $k$ and offset by its initial value $\eta_{0}$. Figure 25 shows our experimental data together with: data from Jacobs $[15,16]$; a Raptor calculation; and three analytical models $[17,18,19]$ that have been proposed in the past to predict the evolution of a single-mode interface. The large scatter in the data point at a normalized time near 6.8 is known to be due to an instrumentation malfunction. The rest of the experimental scatter is acceptable for data from a large facility like 


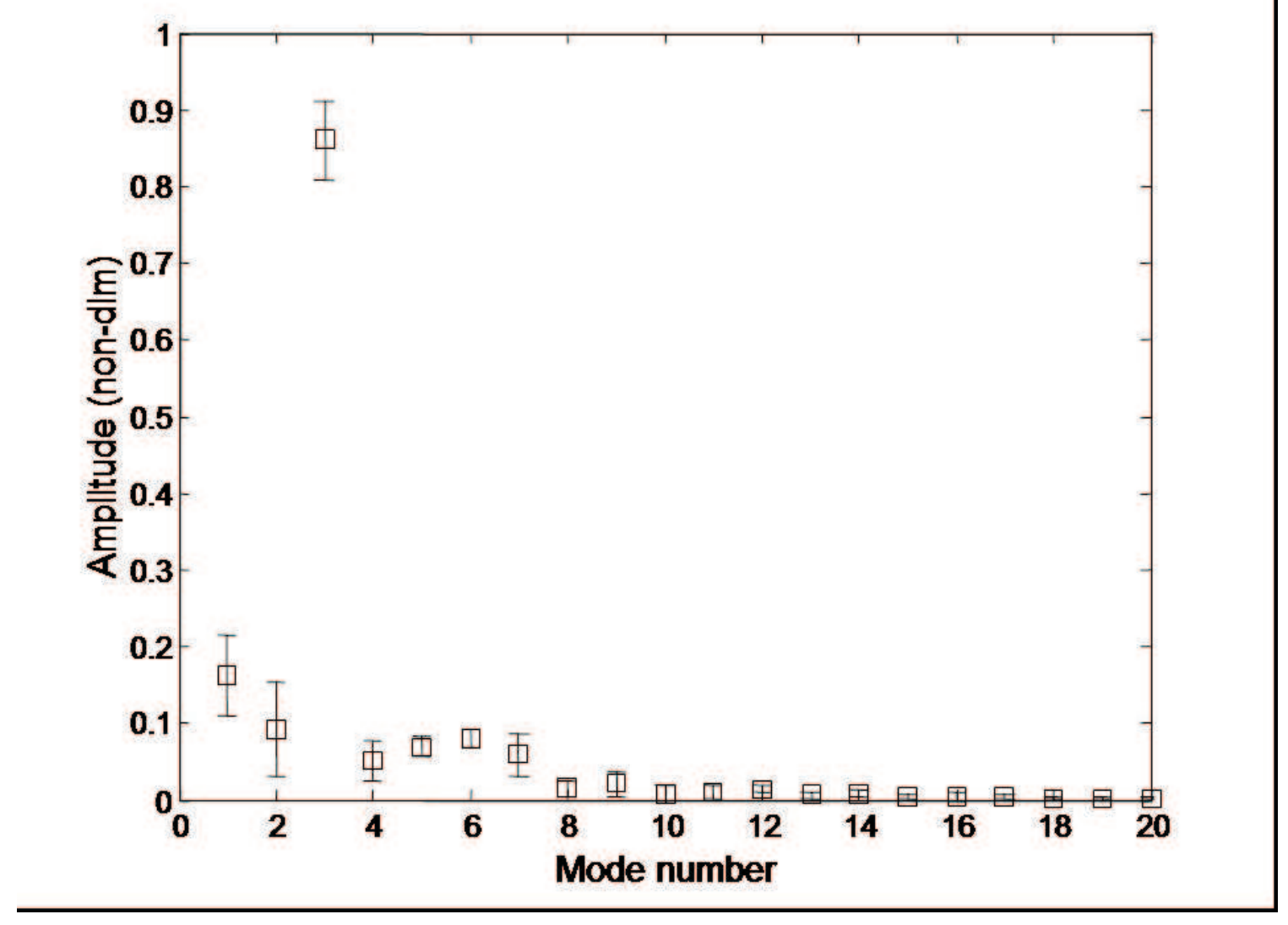

Figure 21: Spectrum of a typical initial condition.

the one used in these experiments. The data indicate that the Sadot model provides a better fit for Jacob's data while Mikaelian's and Di Monte's models adequately describe the present results.

The most important achievements of this portion of the program have been the development of the oscillating piston system for the preparation of an interface and the performance of experiments at Mach numbers greater than 2.

\subsection{Rayleigh-Taylor instability}

\subsubsection{Background}

The investigation of the RTI has followed the same history as that of the RMI, starting from the

single-mode, linear behavior. At present, the main issues being investigated worldwide are the same as those mentioned in Sec. 3.2.1. 


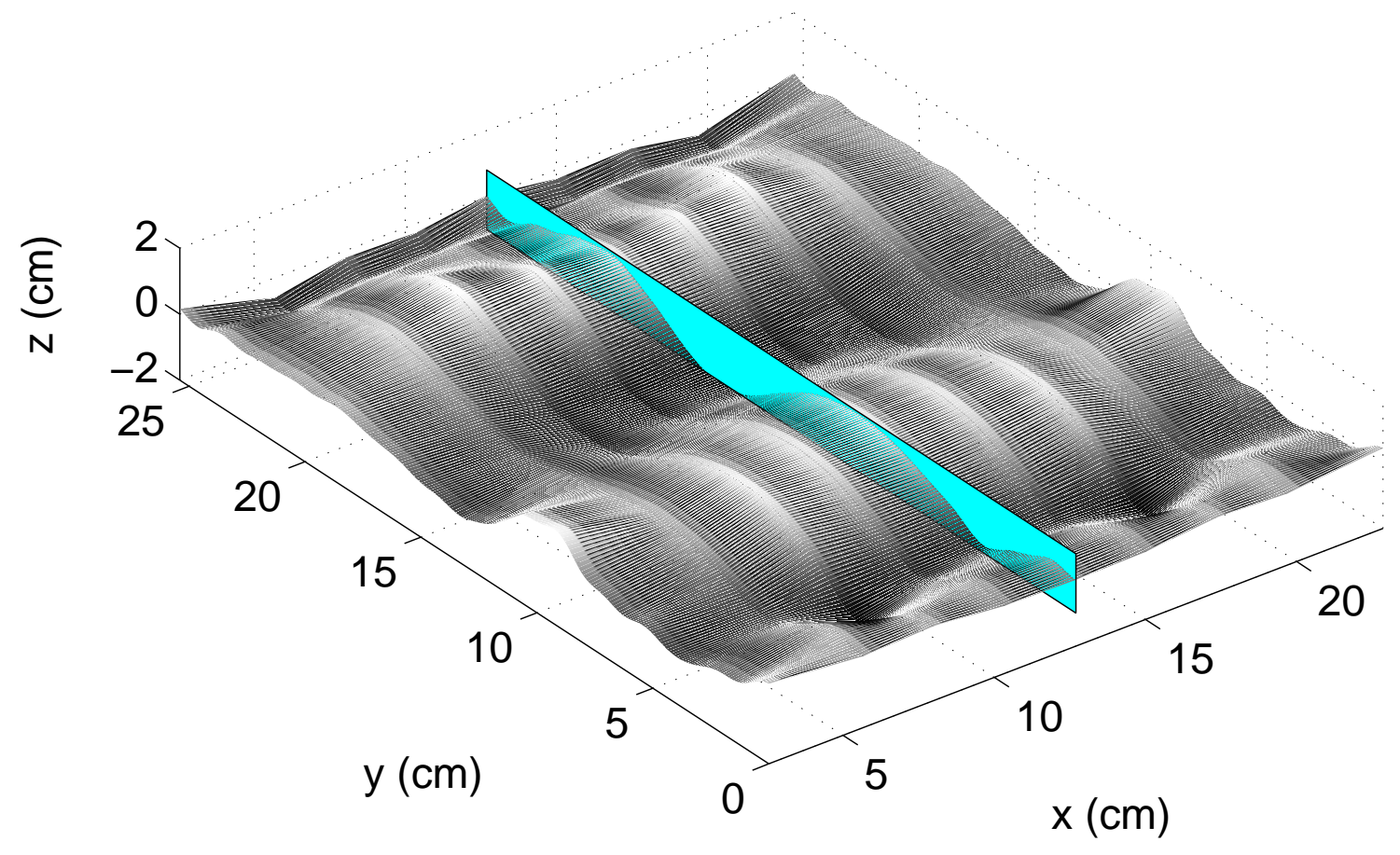

Figure 22: 3-D reconstruction of a typical initial condition.

\subsubsection{Experimental setup}

A new experiment has been developed for the study of the RT instability, based on the use of a magnetorheological (MR) fluid as one of the two fluids at a perturbed interface. MR-fluids are suspension of Fe particles in a solvent, at concentrations large enough that the application of a sufficiently large external magnetic field raises the shear modulus of the suspension to values so high that the MR-fluid effectively "freezes". Thus, an arbitrary shape can be imposed on the free surface of an MR fluid, and the "frozen" MR fluid can be coupled with a different fluid (e.g. water) to form a pair of fluids of different densities separated by a perturbed interface; when the magnetic field is removed, gravity drives the RTI. Use of an MR fluid thus allows for the preparation of interfaces of any desired shape (in particular, with any superposition of sinusoidal modes) and ensures that the experiment begins with both fluids at rest.

By changing the concentration of the Fe particles and the second fluid at the interface, the viscosity of the MR-fluid and the pair's Atwood number can be varied. In selecting the optimal MR fluid composition and fluid pair, the main challenges are: i) maintaining the viscosity of the MR-fluid as low as possible, so as to achieve as high as possible a Reynolds number during the experiment; ii) prevent agglomeration of the Fe particles and leakage of the solvent into the other fluid; iii) ensure that the two fluids are miscible, so as to be able to observe and quantify the transition from a distorted interface to a mixing layer. 


\section{Experiment}
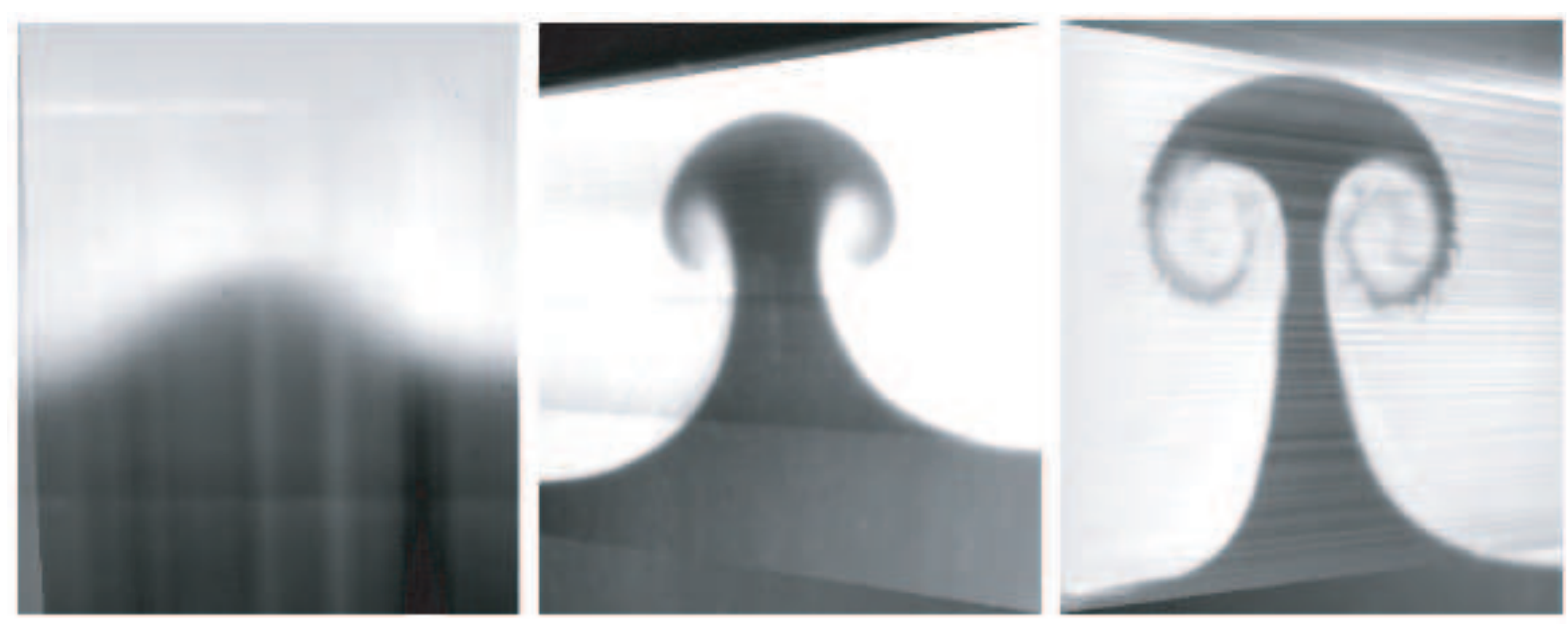

\section{Raptor}
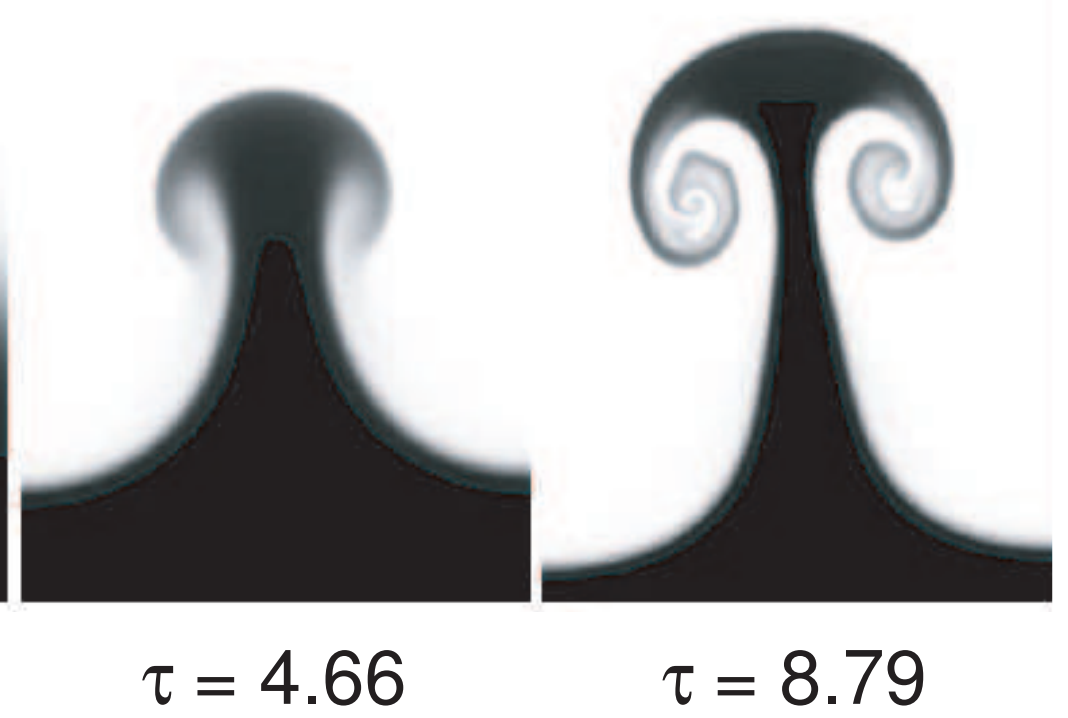

Figure 23: RMI laboratory (top) and computational (bottom) experiments. $\mathrm{N}_{2} / \mathrm{SF}_{6}$ interface; $M=1.3$.

The experimental setup has evolved through several different designs all centered around a plexiglas test section where the interface is initially held in place by the action of two sets of nine $1.5 \mathrm{~T}$ permanent magnets mounted in two magazines. At the beginning of each experiment, the magazines are pulled away from their closest location to the test section by two gas cylinders. The interface is illuminated with diffuse, white light and it is imaged using a $512 \times 512$ pixel CCD camera, operating at $230 \mathrm{fps}$. Views of the apparatus are shown in Fig. 26. Since the contrast between the MR-fluid and the water is very large, the interface shape is easily identified with an edge-detection algorithm based on pixel value.

A large part of our work has been dedicated to identifying the best combination of Fe particles concentration, solvent and pairing fluid. Initially, the MR-fluid used was a dispersion of Fe particles (average diameter $4.5 \mu \mathrm{m}, 15 \%$ volume fraction) in mineral oil, with a small addition of a surfactant (oleic acid) to prevent coalescence of the Fe particles. The resulting Atwood number when that 


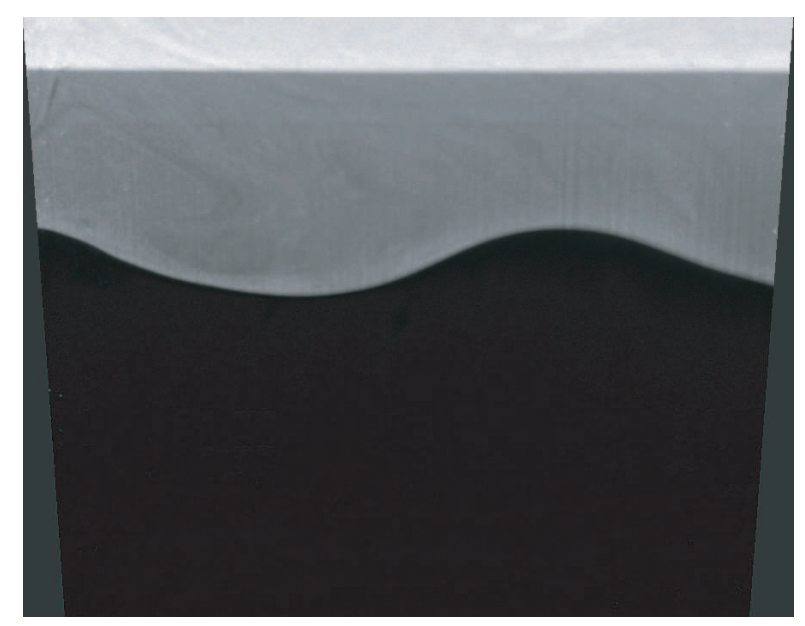

$$
\tau=0.00
$$

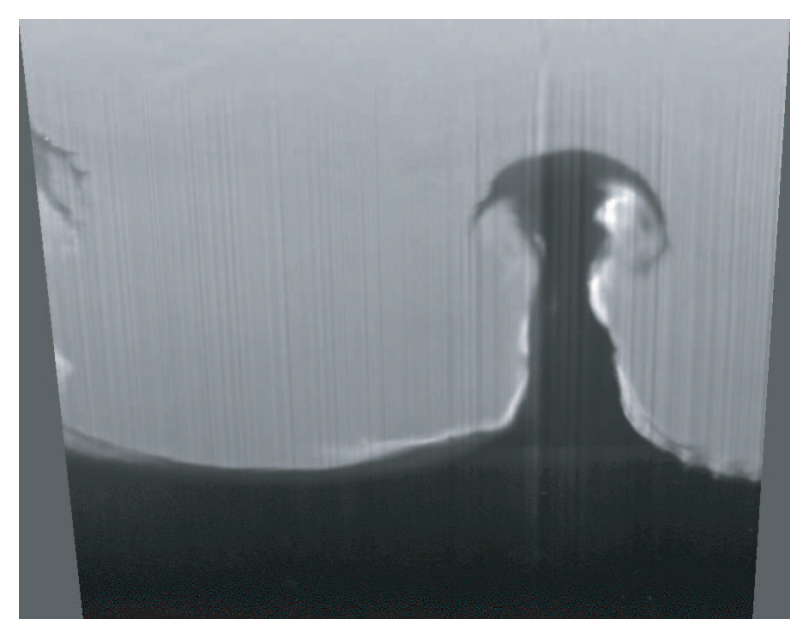

$$
\tau=3.29
$$

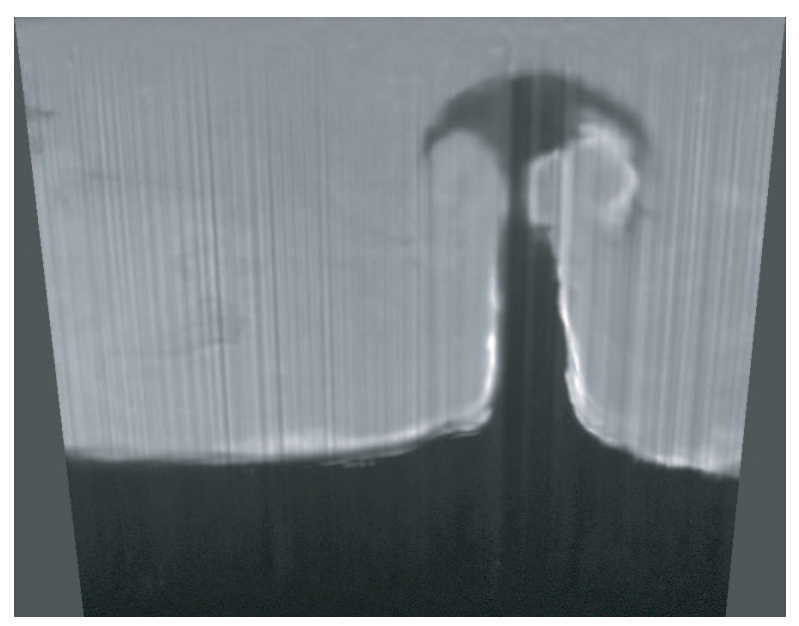

$$
\tau=4.16
$$

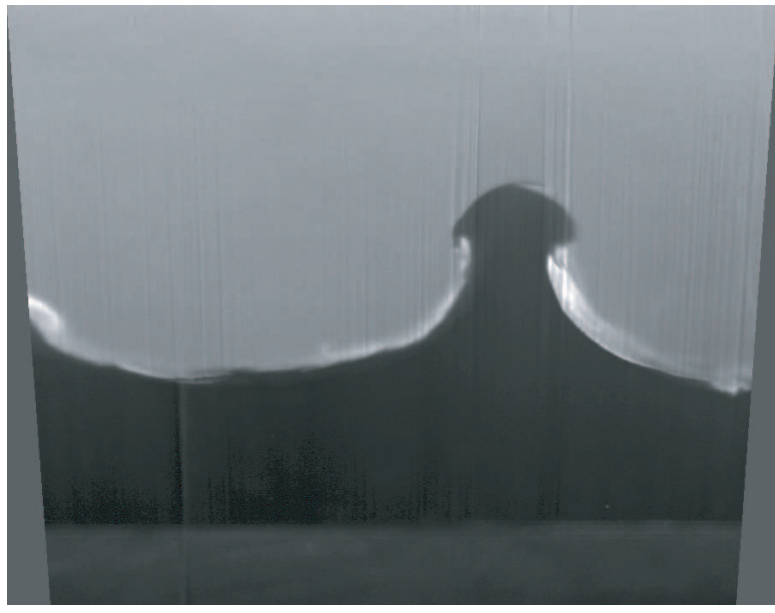

$$
\tau=1.54
$$

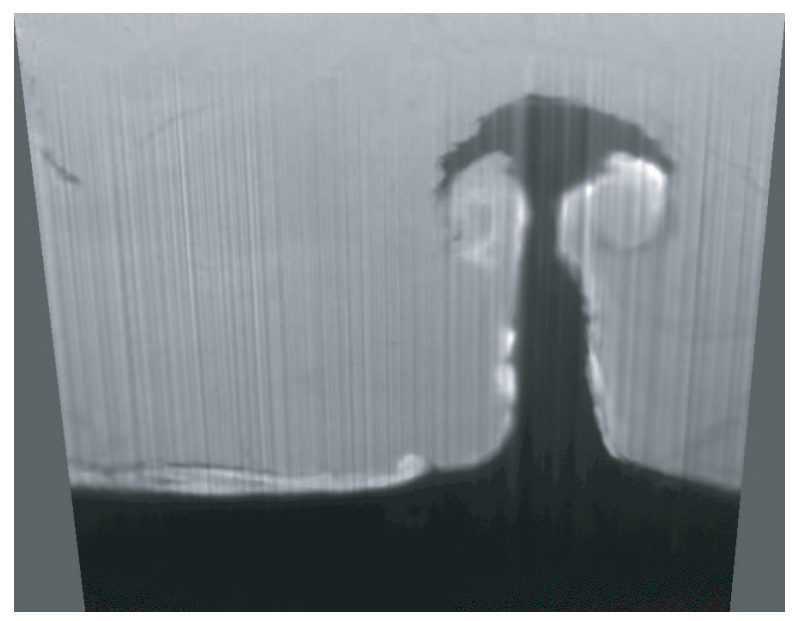

$$
\tau=3.98
$$

Figure 24: RMI laboratory experiments. $\mathrm{N}_{2} / \mathrm{SF}_{6}$ interface; $M=2.05$. 


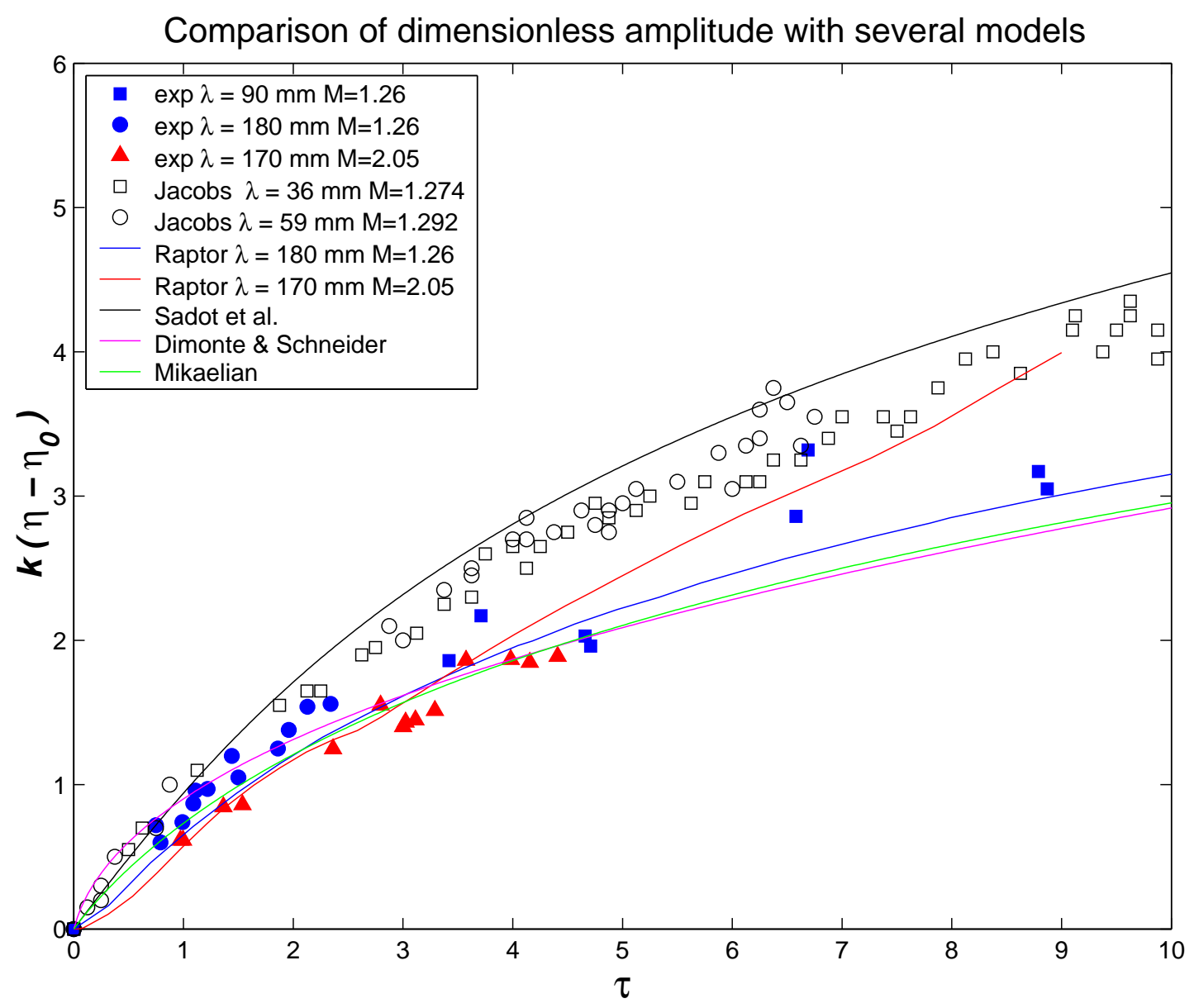

Figure 25: Experimental amplitudes and predictive analytical models.

MR-fluid is paired with water is $A=0.315$. More recently, methanol and hexane have successfully been used as solvents and paired with water, yielding higher Reynolds numbers (maximum value reached so far is around 2000) than when using mineral oil. Methanol-based MR-fluids are miscible with water, making their use at once more challenging and more interesting in the study of RayleighTaylor-driven mixing.

\subsubsection{Results}

Like in the case of the RMI, these experiments aim at measuring the growth rate of the perturbations well into the nonlinear regime, the modal interactions and the mixing rates between the different fluids. This last objective is the hardest to achieve as it requires pairs of miscible fluids.

Sample images of the evolution of a 2-D interface between a mineral oil-based MR-fluid and water, with modes 3 and 5 initially imposed on it are are shown in Fig. 27 and the experimentally-measured evolution of the amplitudes of the first ten modes are shown in Fig. 28.

Samples of experiments performed with a mineral oil-based MR-fluid/water 3-D interface (a hemi- 


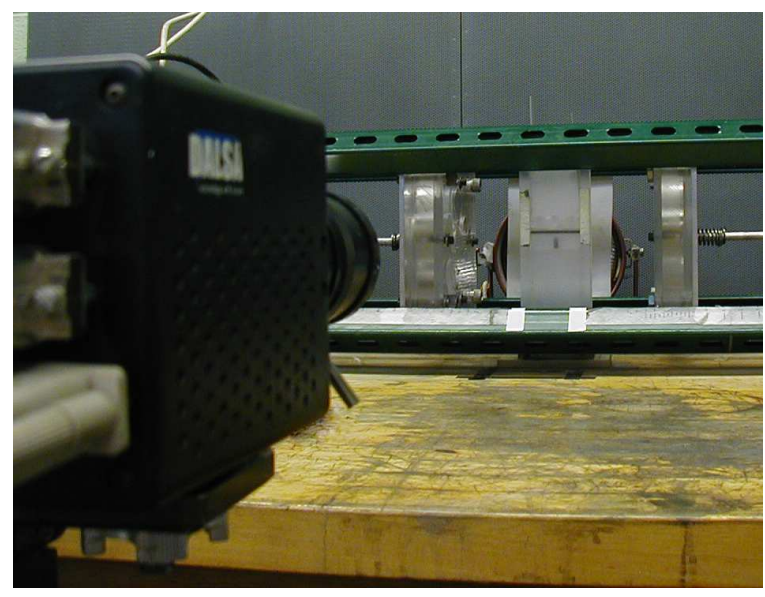

(a)

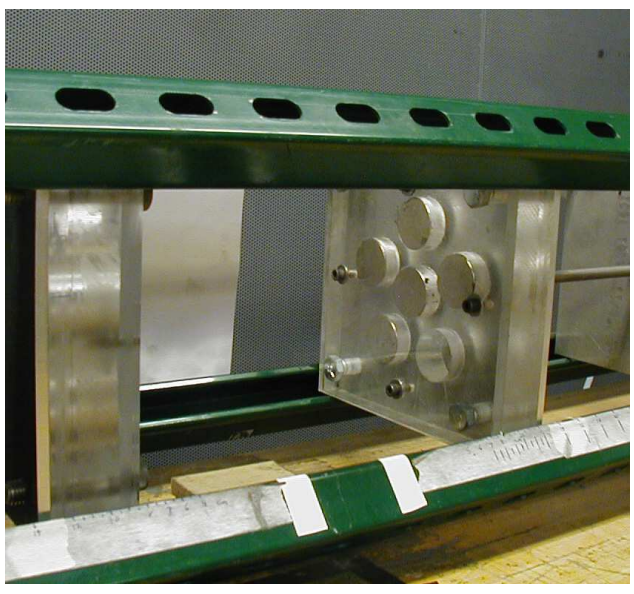

(b)

Figure 26: Views of setup for RT experiments: (a) imaging CCD, test section and magnet magazines; (b) detail of magnet magazine.

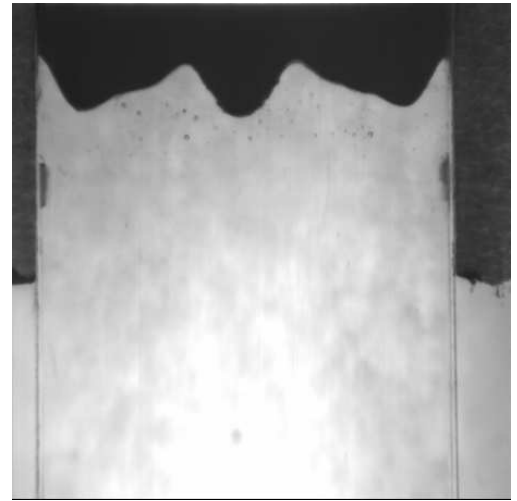

(a)

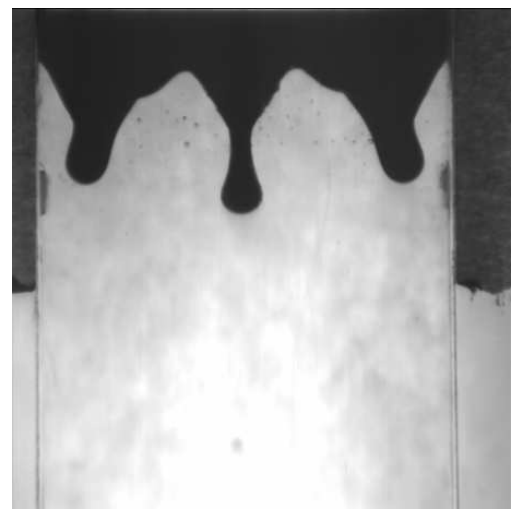

(d)

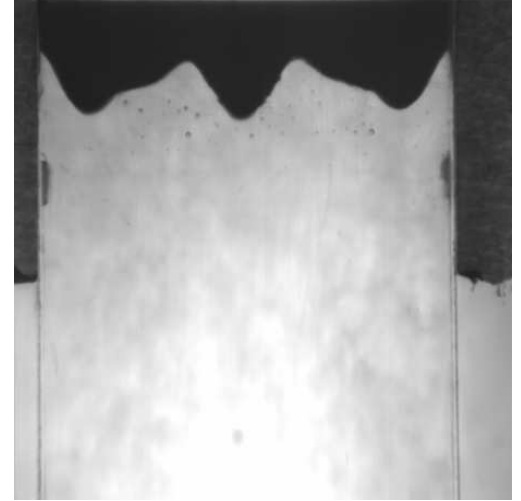

(b)

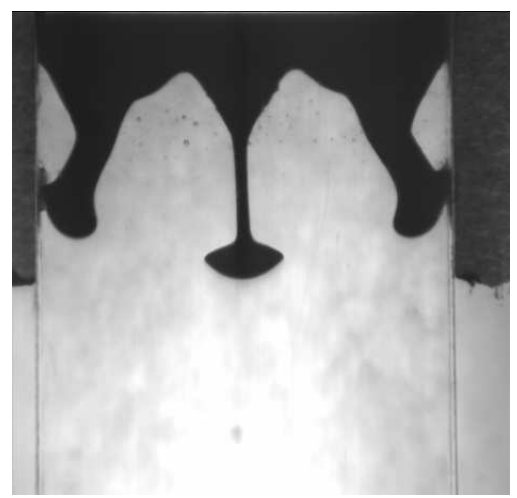

(e)

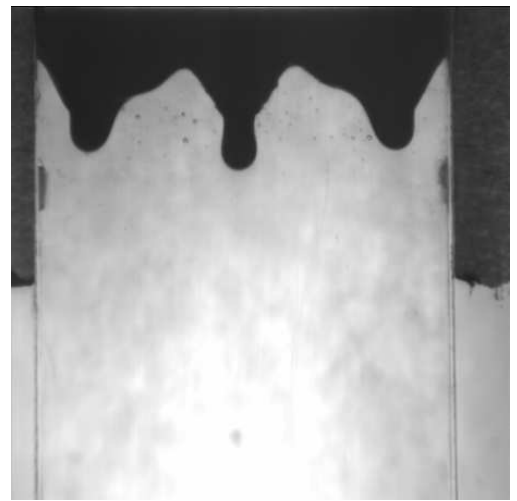

(c)

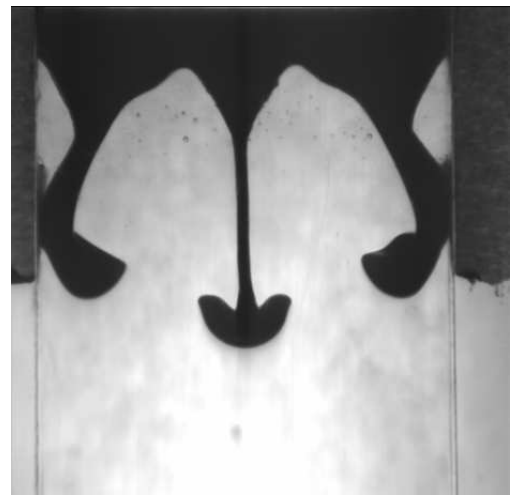

(f)

Figure 27: Images of a multimode MR fluid/water interface. $\phi=20 \%, A=0.35$. (a) 0 s, (b) 92 ms, (c) $168 \mathrm{~ms}$, (d) $206 \mathrm{~ms}$, (e) $264 \mathrm{~ms}$, (f) $321 \mathrm{~ms}$. 


\section{Normalized Modal Growth}

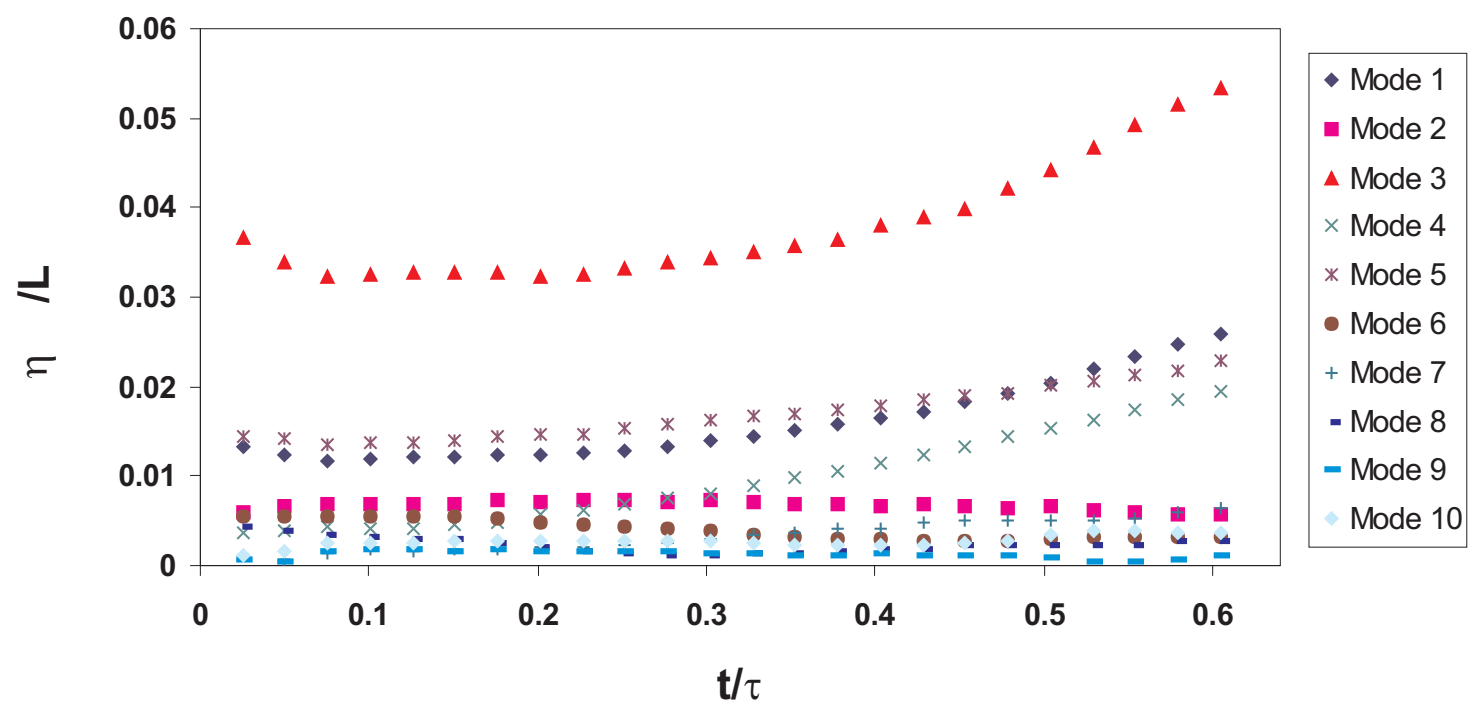

Figure 28: Modal growth on a 2-modes (3 and 5) interface.

sphere) are shown in Fig. 29.

The use of MR-fluids to form an initial condition for an RTI experiment is an innovative concept never tried before for this purpose. The main achievements of this part of the program have been: the entire development of these new experiments; the identification of optimal MR-fluid composition and fluid pairing; the performance of the first experiments based on this novel technique leading to measurements of interface amplitude well into the non-linear regime. 


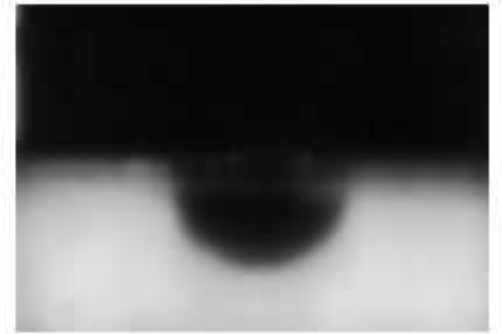

(a)

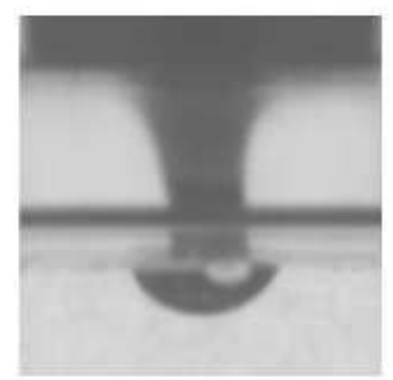

(c)

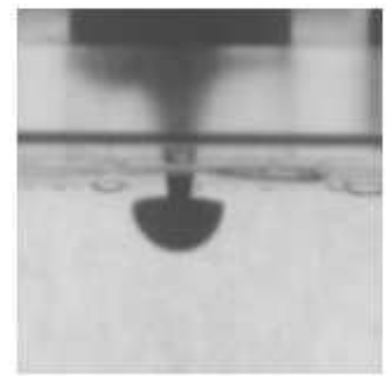

(e)

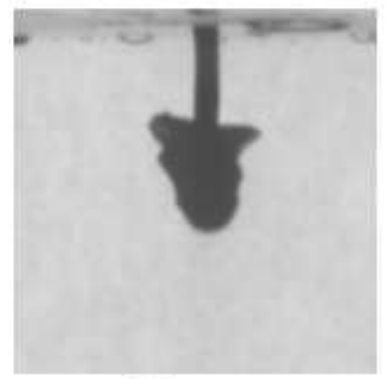

(g)

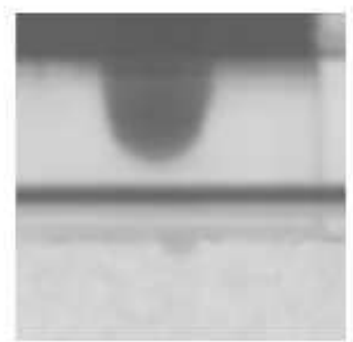

(b)

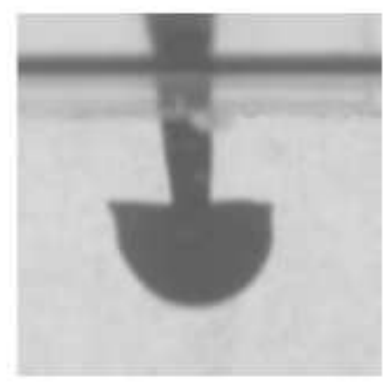

(d)

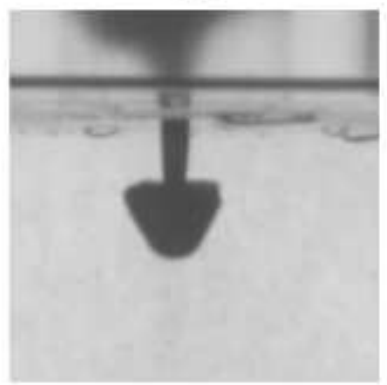

(f)

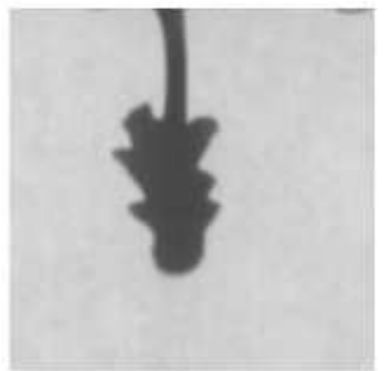

(h)

Figure 29: Evolution of an MR-fluid hemisphere paired with water.

\section{References}

[1] J.-F. Haas and B. Sturtevant. Interaction of weak shock waves with cylindrical and spherical inhomogeneities. J. Fluid Mech., 181:41-76, 1987. 
[2] R. D. Cohen. Shattering of a liquid drop due to impact. Proc. R. Soc. Lond. A, 435:483-503, 1991.

[3] D. Ranjan, M. H. Anderson, J. G. Oakley, and R. Bonazza. Experimental investigation of a strongly shocked gas bubble. Phys. Rev. Lett., 94(184507), 2005.

[4] N.J. Zabusky and S.M. Zeng. Shock cavity implosion morphologies and vortical projectile generation in axisymmetric shock-spherical fast/slow bubble interactions. J. Fluid Mech., 362:327-346, 1998.

[5] S. E. Widnall, D. B. Bliss, and C. Y. Tsai. The instability of short waves on a vortex ring. J. Fluid Mech., 66:35, 1974.

[6] S. K. Godunov. A difference method for numerical calculation of discontinuous solutions of the equations of hydrodynamics. Mat. Sb., 47(89):271-306, 1959.

[7] P. Colella. A direct Eulerian MUSCL scheme for gas dynamics. SIAM J. Sci. Stat. Comput., 6(1):104-117, 1985.

[8] M. Berger and J. Oliger. Adaptive mesh refinement for hyperbolic partial differential equations. J. Comput. Phys., 53:484-512, 1984.

[9] M. Berger and P. Colella. Local adaptive mesh refinement for shock hydrodynamics. J. Comput. Phys., 82(1), 1989.

[10] G. Layes, G. Jourdan, and L. Houas. Distortion of a spherical gaseous interface accelerated by a plane shock wave. Phys. Rev. Lett., 91(17), 2003.

[11] G. Layes, G. Jourdan, and L. Houas. Experimental investigation of the shock wave interaction with a spherical gas inhomogeneity. Phys. Fluids, 17(028103), 2005.

[12] D. Ranjan, J. Niederhaus, M. H. Anderson, B. Motl, J. G. Oakley, J. Greenough, and R. Bonazza. Experimental study of the interaction of a planar shock with a free rising bubble. In Proceedings of the Tenth International Workshop on Compressible Turbulent Mixing, 2006.

[13] J. Giordano and Y. Burtschell. Richtmyer-Meshkov instability induced by shock-bubble interaction: numerical and analytical studies with experimental validation. Phys. Fluids, 18(036102), 2006.

[14] Ye.Ye. Meshkov. Instability of a shock wave accelerated interface between two gases. NASA Technical Translation F-13,074, 1970.

[15] M.A. Jones and J.W. Jacobs. A membraneless experiment for the study of the RichtmyerMeshkov instability of a shock-accelerated gas interface. Phys. Fluids 9(10) 3078-3085, 1997.

[16] J.W. Jacobs and V.V. Krivets. Experiments on the late-time development of a single-mode Richtmyer-Meshkov instability. Physics of Fluids 17(034105), 2005.

[17] O. Sadot, L. Erez, U. Alon, D. Oron, L. Levin, G. Erez, G. Ben-Dor, and D. Shvarts. Study of nonlinear evolution of single-mode and two-bubble interaction under Richtmyer-Meshkov instability. Phys. Rev. Lett., 80, 1654-1657, 1998. 
[18] G. Dimonte and M. Schneider. Density ratio dependence of Rayleigh-Taylor mixing for sustained and impulsive acceleration histories. Phys. of Fluids 12, 2000.

[19] K. Mikaelian. Explicit expressions for the evolution of single-mode Rayleigh-Taylor and Richtmyer-Meshkov instabilities at arbitrary Atwood numbers. Phys. Rev. E, 67, 0263191-026319-7, 2003. 


\section{Publications and presentations}

\section{Journal articles}

Niederhaus, J.H.J., Greenough J.A., Oakley J.G., Ranjan D., Anderson M.H., and Bonazza R., A Computational Paramater Study for the Three-Dimensional Shock-Bubble Interaction, to be submitted to the Journal of Fluid Mechanics, November, 2006

Ranjan D., Niederhaus J., Motl B., Oakley J., Anderson M., Bonazza R., Experimental investigation of primary and secondary features in high-Mach-number shock-bubble interaction, in review Phys. Rev. Lett.

Ranjan D., Anderson M., Oakley J., Bonazza R., Experimental investigation of a strongly shocked gas bubble, Phys. Rev. Lett. 94, 184507, 2005

\section{Conference papers}

White J., Oakley J., Anderson M., Bonazza R., The Rayleigh-Taylor Instability at a Water/Magnetorheological Fluid Interface, Proceedings of the 10th International Workshop on the Physics of Compressible Turbulent Mixing, Paris, France, July 2006.

Niederhaus J., Oakley J., Ranjan D., Anderson M., Bonazza R., Greenough J., A computational parameter study for the 3D shock-bubble interaction, with and without modeled soap film, Proceedings of the 10th International Workshop on the Physics of Compressible Turbulent Mixing, Paris, France, July 2006.

Ranjan D., Niederhaus J., Anderson M., Motl B., Oakley J., Bonazza R., Greenough J., Experimental study of the interaction of a planar shock with a free rising bubble, Proceedings of the 10th International Workshop on the Physics of Compressible Turbulent Mixing, Paris, France, July 2006.

Motl B., Niederhaus J., Oakley J., Ranjan D., Anderson M., Bonazza R., Shock accelerated twodimensional interface, Proceedings of the 10th International Workshop on the Physics of Compressible Turbulent Mixing, Paris, France, July 2006.

Ranjan D., Niederhaus J.H.J., Oakley J.G., Anderson M.H., Greenough J.A., Bonazza R., Shockinduced instabilities on a spherical gas bubble, Proceedings of the 25th International Symposium on Shock Waves, Bangalore, India, July 2005

Motl B.J., Niederhaus J.H.J., Anderson M.H., Oakley J.G., Bonazza R. Richtmyer-Meshkov instability of a membraneless, sinusoidal gas interface, Proceedings of the 25th International Symposium on Shock Waves, Bangalore, India, July 2005

\section{Conference abstracts}

Motl B., Niederhaus J., Anderson M., Oakley J., Bonazza R., Richtmyer-Meshkov instability of a membraneless, sinusoidal gas interface, 58th Annual Meeting of APS Division of Fluid Dynamics, Chicago, November 2005

White J., Anderson M., Oakley J., Bonazza R., Study of the Rayleigh-Taylor instability at a hemispherical interface using a magnetorheological fluid, 58th Annual Meeting of APS Division of Fluid Dynamics, Chicago, November 2005

Ranjan D., Niederhaus J., Anderson M., Oakley J., Bonazza R., Interaction of a planar shock wave with a spherical gas inhomogeneity. Part I: Experiments, 58th Annual Meeting of APS Division of 
Fluid Dynamics, Chicago, November 2005

Niederhaus J., Ranjan D., Anderson M., Oakley J., Bonazza R., Interaction of a planar shock wave with a spherical gas inhomogeneity. Part II: Calculations, 58th Annual Meeting of APS Division of Fluid Dynamics, Chicago, November 2005 


\section{Personnel}

Faculty and staff who have been involved in and supported by the program, for its entire duration or parts of it:

Prof. Riccardo Bonazza, Associate Professor, Dept. Engineering Physics, PI.

Prof. Leslie Smith, Professor, Depts. Mathematics and Engineering Physics.

Dr. Mark Anderson, Associate Scientist, Dept. Engineering Physics.

Dr. Jason Oakley, Assistant Scientist, Dept. Engineering Physics.

Mr. Paul Brooks, Instrumentation Specialist, Dept. Engineering Physics.

Graduate students supported by and fully involved in the program in the past three years include:

Mr. Brad Motl (USA; pursuing a Ph.D. degree).

Mr. John Niederhaus (USA; pursuing a Ph.D. degree).

Mr. Jeremy White (USA; pursuing a Ph.D. degree).

Mr. Devesh Ranjan (India; pursuing a Ph.D. degree).

Mr. Alec Bates (USA; completed a Master's degree).

Mr. Chaine Selig (USA; completed a Master's degree).

Nine undergraduate students were also involved in and supported by the program at various levels and for different lengths of time. 